\title{
Polarization gating based on Mueller matrices
}

\author{
Angel Lizana \\ Albert Van Eeckhout \\ Kamil Adamczyk \\ Carla Rodríguez \\ Juan Carlos Escalera \\ Enric Garcia-Caurel \\ Ignacio Moreno \\ Juan Campos
}




\title{
Polarization gating based on Mueller matrices
}

\author{
Angel Lizana, ${ }^{\mathrm{a}, \star}$ Albert Van Eeckhout, ${ }^{\mathrm{a}}$ Kamil Adamczyk, ${ }^{\text {a }}$ Carla Rodríguez, ${ }^{\mathrm{a}}$ Juan Carlos Escalera, ${ }^{\mathrm{a}}$ \\ Enric Garcia-Caurel, ${ }^{\mathrm{b}}$ Ignacio Moreno, ${ }^{\mathrm{c}}$ and Juan Campos ${ }^{\mathrm{a}}$ \\ aUniversitat Autònoma de Barcelona, Grup d'Òptica, Physics Department, Bellaterra, Spain \\ bUniversité Paris-Saclay, LPICM, CNRS, École Polytechnique, Palaiseau, France \\ 'Universidad Miguel Hernández, Department of Materials Science, Optics and Electronics Technology, Elche, Spain
}

\begin{abstract}
We present mathematical formulas generalizing polarization gating (PG) techniques. PG refers to a collection of imaging methods based on the combination of different controlled polarization channels. In particular, we show how using the measured Mueller matrix (MM) of a sample, a widespread number of PG configurations can be evaluated just from analytical expressions based on the MM coefficients. We also show the interest of controlling the helicity of the states of polarization used for PG-based metrology, as this parameter has an impact in the image contrast of samples. In addition, we highlight the interest of combining PG techniques with tools of data analysis related to the MM formalism, such as the well-known MM decompositions. The method discussed in this work is illustrated with the results of polarimetric measurements done on artificial phantoms and real ex-vivo tissues. $\odot 2017$ Society of Photo-Optical Instrumentation Engineers (SPIE) [DOI: 10.1117/1.JBO.22.5.056004]
\end{abstract}

Keywords: polarization gating; Mueller matrix; biological tissue; polarimetric image contrast.

Paper 170020R received Jan. 13, 2017; accepted for publication Apr. 17, 2017; published online May 10, 2017.

\section{Introduction}

In the last decade, polarimetric information has proven to be useful for biological tissues inspection. ${ }^{1-3}$ As a result, polarimetric-based techniques are nowadays being applied in multiple scenarios, such as cancer detection and stage identification, ${ }^{4-8}$ to enhance image contrast in skin diseases, ${ }^{9-11}$ for human eye examination, ${ }^{12,13}$ for diabetes diagnosis as well as therapy, ${ }^{14,15}$ etc.

There are different ways to extract sample information from polarimetric measurements (such as surface roughness, tissue spatial inhomogeneities, biological material recognition, optical properties of turbid tissues, tissue depth metrology, subsurface examination, etc.), as well as to enhance the contrast of images taken from the sample. A widespread polarimetric technique applied for biological tissues inspection is the so-called polarization gating (PG). ${ }^{16-20}$ Essentially, PG techniques exploit the fact that samples respond differently depending on the polarization state of the light used to probe it. The basic PG configuration consists of using linear polarization for illumination and detection [linear polarization gating (LPG)] stage. Then, LPG images can be combined to provide a new processed image, for instance, by subtracting the images captured with parallel and with crossed polarizers. To further improve image contrast, recent studies have provided the interest of generalizing LPG techniques with the use of elliptical polarization, i.e., elliptical polarization gating (EPG). ${ }^{17,19}$

Another group of polarimetric methods is based on the measurement of the Mueller matrix (MM) of the sample, and the subsequent analysis of the polarimetric content which is encoded in the corresponding 16 real MM coefficients. ${ }^{21}$ These techniques, which provide, in principle, a different polarimetric approach than those given by PG techniques, are used by a number of authors for biological tissues inspection. .7, $, 12,13^{2}$

*Address all correspondence to: Angel Lizana, E-mail: angel.lizana@uab.es
In this paper, we demonstrate that information provided by PG techniques can be alternatively obtained from the experimental MM of the sample. In particular, we derive an analytical expression, which consists of a linear combination of different Mueller coefficients, and we show how a set of PG configurations can be considered as particular cases of the derived analytical expression. The desired PG configuration is then obtained by properly tuning some control parameters, such as the azimuth or the ellipticity of the input and analyzing states of polarization. In addition, we highlight the interest of using MM measurements instead of particular PG configurations. First, due to the MM-based analytical expression, we show in this work that we can extract the polarimetric information of a sample corresponding to multiple PG configurations without the necessity of experimentally measuring each one of them. Instead of this, only the measurement of the experimental MM of the sample is required with our method. Second, Mueller measurements not only allow building a more general analytical expression, but also give access to extra channels of data processing, such as those provided by the different product MM decompositions schemes ${ }^{21-23}$ or even further, by applying recently developed methods such as those based on the differential MM. ${ }^{21,23-26}$

The method proposed in this paper is experimentally tested by using an imaging MM polarimeter based on liquid crystal panels. This allows the system to dynamically perform the experimental measurements without the necessity of mechanical movements. The commutation rate of the used liquid crystal panels is $\sim 60 \mathrm{~Hz}$, and the exposure time of the CCD camera that is used to register the intensity images is adapted for each sample analyzed to capture the maximum intensity without saturating the camera (the exposure time range used for measurements is between 0.004 and $0.12 \mathrm{~s}$ ). With this experimental configuration, full polarimetric measurements can be done in 
few ( 0.4 to 2.2$)$ seconds, which may be of interest in real-time applications. Indeed, if the methods discussed in this manuscript would be considered to be applied for real-time applications, the experimental set-up could be further optimized by using ferroelectric liquid crystal displays or photoelastic modulators. ${ }^{27,28}$ Another interesting application for the methods discussed here is standard scanning microscopy adapted to polarimetric metrology. If these latter applications should be performed in real time, then the high-speed imaging Mueller polarimeter provided in Ref. 29 can be used, which works at the time scale of a scanning microscope.

In a sense, we think that the methods proposed in this work bring together two mathematical tools for polarimetric sample analysis (PG- and MM-based methods) that traditionally have been unlinked and may be of interest in biological applications. ${ }^{16-20,30-32}$

The outline of this manuscript is as follows. In Sec. 2, we first briefly describe some of the more commonly used PG configurations (Sec. 2.1). Afterward, in Sec. 2.2, we derive a general expression based on the MM coefficients of the studied sample, from which the different PG configurations described in Sec. 2.1 become individual solutions. In particular, each different PG configuration is achieved from the proposed general expression by properly tuning two control parameters in the mathematical equation, representing the azimuth and ellipticity values of the polarizations illuminating and analyzing the sample. In Sec. 3, we describe the optical scheme that is used to measure the MM of different samples, from which different PG configurations are achieved (Sec. 3.1). In addition, the description of the different samples inspected in this work is also provided (Sec. 3.2). In Sec. 4, we provide the equivalence between standard PG and PG configurations obtained from MM metrology (Sec. 4.1). Next, in Sec. 4.2, we highlight the interest of combining PG configurations with well-known MM analytical tools. Finally, the main conclusions of the work are given in Sec. 5 .

\section{Polarization Gating Configurations Derived from Mueller Matrix Data}

In this section, we briefly describe some PG configurations currently used for tissue inspection and we discuss some of the strengths and drawbacks related to them (Sec. 2.1). The nomenclature used to label the different PG configurations described in Sec. 2.1 is that followed in Ref. 19. Afterward, we derive an analytical expression that consists of a linear combination of different functions which depend on several of the MM coefficients of the measured sample (Sec. 2.2). Note that such a relation can be interpreted as a generalization of multiple PG configurations.

\subsection{Polarization Gating Techniques}

When polarized light interacts with biological tissues, the characteristics of the produced scattered light strongly depend on different parameters, such as the sample molecules' size, the polarization of the input light, the wavelength, and the sample depth proven, among others.

One typical measuring configuration (let us call it configuration $\mathrm{C} 1$ ) consists of illuminating the analyzed sample with linear polarization and projecting the scattered light to the same input polarization (i.e., parallel polarizer-analyzer). When illuminating the sample with a linear polarization, a mixed contribution of different kind of photons is observed when measuring out of the ballistic direction. ${ }^{19,33}$ We can subgroup them as photons reflected at the tissue surface (surface-reflected photons, $\mathrm{S}_{\mathrm{L}}$ ), photons penetrating to the subsurface but maintaining the original polarization (polarization maintaining photons, $\mathrm{P}_{\mathrm{L}}$ ), and photons reaching deeper layers of the sample, which are fully depolarized by multiple scattering events (depolarized photons, $\mathrm{D}_{\mathrm{L}}$ ).

Therefore, the $\mathrm{C} 1$ configuration leads to a mixture of all three kinds of $\mathrm{S}_{\mathrm{L}}, \mathrm{P}_{\mathrm{L}}$, and $\mathrm{D}_{\mathrm{L}}$ photon contributions. However, as $\mathrm{P}_{\mathrm{L}}$ photons are those that usually provide the most significant information of the studied sample, different strategies can be applied to remove the image background and to improve the image contrast, by eliminating the nondesired $\mathrm{S}_{\mathrm{L}}$ and $\mathrm{D}_{\mathrm{L}}$ contributions as much as possible. To this aim, some authors have proposed to combine the $\mathrm{C} 1$ configuration with a second measurement, where the sample is illuminated with linear polarized light and scattered light is imaged through the corresponding orthogonal linear polarization (cross linear configuration, here labeled as $\mathrm{C} 2$ ). Images recorded by using the $\mathrm{C} 2$ configuration (crossed polarizers) are mainly based on $\mathrm{D}_{\mathrm{L}}$ photons, because $\mathrm{P}_{\mathrm{L}}$ and $\mathrm{S}_{\mathrm{L}}$ photons are efficiently filtered by the linear analyzer. ${ }^{19}$ Under this scenario, by simply obtaining the difference, C1C2 (let us call this PG configuration as linear configuration, LConfig), we obtain polarimetric images which are only due to $\mathrm{P}_{\mathrm{L}}$ and $\mathrm{S}_{\mathrm{L}}$ photons $\left(\mathrm{D}_{\mathrm{L}}\right.$ photons are removed), thus, the image quality is improved when compared with the $\mathrm{C} 1$ configuration by itself. However, by using the LConfig, the contribution of SL photons still degrades the image contrast to a certain extent.

To improve the final image, recent studies have suggested an interest in generalizing LPG with the use of elliptical polarization, i.e., EPG. ${ }^{19,33-35}$ In particular, EPG presents two main benefits when compared with LPG. First, elliptical states change the sense of rotation by surface reflection, thus, specular reflection is eliminated by using a coelliptical configuration for detection. Second, elliptically polarized light holds its polarization state for a larger number of scattering events than linearly polarized light, so it is suitable for the study of sub-substrate structures. ${ }^{19}$ In particular, when illuminating a sample with an elliptical polarization out of the ballistic direction, a mixed contribution of photons (elliptical polarization maintaining photons, $\mathrm{P}_{\mathrm{E}}$ and depolarized photons, $\mathrm{D}_{\mathrm{E}}$ ) is observed for a coelliptical configuration (let us call it configuration $\mathrm{C} 3$ ). ${ }^{19,33}$ Note that by using the coelliptical configuration, $S_{E}$ photons are removed because they undergo a change in helicity by reflection. ${ }^{19,35}$ On the other hand, by using a cross-elliptical configuration (i.e., illuminating with a given elliptical polarization and detecting with the orthogonal one; configuration $\mathrm{C} 4$ ), a mix of surface-reflected elliptical photons, $S_{E}$, and $D_{E}$ photons, is obtained, as $\mathrm{P}_{\mathrm{E}}$ photons are removed in the cross-elliptical configuration. ${ }^{19,35}$ Thus, analogously to the LPG case (LConfig), by simply subtracting the $\mathrm{C} 3$ and $\mathrm{C} 4$ channels (i.e., $\mathrm{C} 3-\mathrm{C} 4$ ), the obtained images are mainly produced by $\mathrm{P}_{\mathrm{E}}$ and $\mathrm{S}_{\mathrm{E}}$ photons, as the nondesired contribution of $\mathrm{D}_{\mathrm{E}}$ photons is removed by subtraction. Let us label this PG configuration (C3-C4) as an elliptical configuration (EConfig). Nevertheless, by using the EConfig, the contribution of $S_{E}$ photons is still present and somewhat degrades the final image contrast.

Other PG approaches can be found in literature to improve the final image contrast. For instance, a clever approximation is derived in Ref. 19. The authors provide an analytical function that combines the above-described [(C1-C2) and (C3-C4)] PG configurations. This leads to a mixture of surface reflected and 
polarization maintaining photons from linear and elliptical polarizations $\left(\mathrm{P}_{\mathrm{L}}+\mathrm{S}_{\mathrm{L}}+\mathrm{P}_{\mathrm{E}}-\mathrm{S}_{\mathrm{E}}\right)$. Note that, in general, the flux for the $S_{L}$ and $S_{E}$ photons is different, because the portion of input light projected to the plane of incidence is different for the linear and elliptical polarization cases. However, by normalizing the amount of reflected light projected in the plane of incidence for the elliptical polarization case to that of the linear polarization case by a factor $\beta$, such that $\mathrm{S}_{\mathrm{E}}=\beta \mathrm{S}_{\mathrm{L}}$, the influence of $S_{L}$ and $S_{E}$ photons in the final processed image can be avoided. In particular, this function, here labeled as function $f$, can be calculated as follows: ${ }^{19}$

$f=(C 3-C 4)+\beta(C 1-C 2)$,

where the numerical parameter $\beta$ corrects the differences in the amount of light projected to the components parallel ( $p$-polarized) and perpendicular ( $s$-polarized) to the plane of incidence when using different ellipticities for the input state of polarization. This parameter $\beta$ has to be experimentally determined for each particular elliptical configuration (C3-C4) applied in the function $f .{ }^{19}$

Note that by using the function $f$, the photons contributing to the final image are mainly those maintaining the polarization, $\mathrm{P}_{\mathrm{L}}$ and $\mathrm{P}_{\mathrm{E}}$ photons, while the background, related to $\mathrm{D}_{\mathrm{L}}, \mathrm{D}_{\mathrm{E}}$, $\mathrm{S}_{\mathrm{E}}$, and $\mathrm{S}_{\mathrm{L}}$ photons, is removed.

\subsection{Polarization Gating Configurations Described Using the Mueller Matrix Formalism}

In this section, we derive an analytical expression which depends on several MM coefficients, that allows performing a number of PG configurations by properly setting a few control parameters. To this aim, we adopt the well-known StokesMueller formalism. ${ }^{21,22}$ Accordingly, the state of polarization of a fully, partial, or unpolarized light beam is described by four intensity values, usually arranged in column-form in the so-called Stokes vector. In turn, the polarimetric response of any sample can be described by its $4 \times 4 \mathrm{MM}$, whose 16 real coefficients encode the polarimetric information of the sample. In this framework, an MM can be understood as the polarization transfer function of the medium and the interaction of light with the medium is described by the following linear system: ${ }^{21,22}$

$S_{\text {out }}=M \cdot S_{\text {input }}=\left(\begin{array}{cccc}m_{00} & m_{01} & m_{02} & m_{03} \\ m_{10} & m_{11} & m_{12} & m_{13} \\ m_{20} & m_{21} & m_{22} & m_{23} \\ m_{30} & m_{31} & m_{32} & m_{33}\end{array}\right) \cdot\left(\begin{array}{c}S_{0} \\ S_{1} \\ S_{2} \\ S_{3}\end{array}\right)$

where $m_{i k}$ are the coefficients of the MM and $S_{i}$ are the different channels of the Stokes vector.

When dealing with fully polarized light beams, the normalized Stokes vector can be written as a function of the polarization angles of the polarization ellipse, i.e., the azimuth $(\alpha)$ and the ellipticity $(\varepsilon)$ angles: $:^{22}$

$S=\left(\begin{array}{c}1 \\ \cos 2 \varepsilon \cos 2 \alpha \\ \cos 2 \varepsilon \sin 2 \alpha \\ \sin 2 \varepsilon\end{array}\right)$

Note that by properly selecting the values for $\alpha$ and $\varepsilon$ in Eq. (3), any fully polarized state of polarization can be described. According to Eqs. (2) and (3), when a fully polarized light beam interacts with a sample, the state of polarization $S_{\text {out }}$ of the output (transmitted, reflected or scattered) light beam can be written as follows:

$S_{\text {out }}=\left(\begin{array}{l}m_{00}+m_{01} \cos 2 \varepsilon \cos 2 \alpha+m_{02} \cos 2 \varepsilon \sin 2 \alpha+m_{03} \sin 2 \varepsilon \\ m_{10}+m_{11} \cos 2 \varepsilon \cos 2 \alpha+m_{12} \cos 2 \varepsilon \sin 2 \alpha+m_{13} \sin 2 \varepsilon \\ m_{20}+m_{21} \cos 2 \varepsilon \cos 2 \alpha+m_{22} \cos 2 \varepsilon \sin 2 \alpha+m_{23} \sin 2 \varepsilon \\ m_{30}+m_{31} \cos 2 \varepsilon \cos 2 \alpha+m_{32} \cos 2 \varepsilon \sin 2 \alpha+m_{33} \sin 2 \varepsilon\end{array}\right)$.

Afterward, we can project (i.e., analyze) this output light beam, $S_{\text {out }}$, on a polarization detector system setting a polarization analyzer (let us call it $S_{\mathrm{PSD}}$ ) equal to the input polarization [i.e., that set in Eq. (3)]. This situation represents a coelliptical measurement and leads to

$$
\begin{aligned}
I_{\text {co-elliptical }}= & S_{\mathrm{PSD}}^{\mathrm{T}} \cdot S_{\mathrm{out}} \\
= & m_{00}+m_{01} \cos 2 \varepsilon \cos 2 \alpha+m_{02} \cos 2 \varepsilon \sin 2 \alpha+m_{03} \sin 2 \varepsilon+m_{10} \cos 2 \varepsilon \cos 2 \alpha+m_{11}(\cos 2 \varepsilon \cos 2 \alpha)^{2} \\
& +m_{12}(\cos 2 \varepsilon)^{2} \cos 2 \alpha \sin 2 \alpha+m_{13} \cos 2 \varepsilon \cos 2 \alpha \sin 2 \varepsilon+m_{20} \cos 2 \varepsilon \sin 2 \alpha+m_{21}(\cos 2 \varepsilon)^{2} \cos 2 \alpha \sin 2 \alpha \\
& +m_{22}(\cos 2 \varepsilon \sin 2 \alpha)^{2}+m_{23} \cos 2 \varepsilon \sin 2 \alpha \sin 2 \varepsilon+m_{30} \sin 2 \varepsilon+m_{31} \cos 2 \varepsilon \cos 2 \alpha \sin 2 \varepsilon \\
& +m_{32} \cos 2 \varepsilon \sin 2 \alpha \sin 2 \varepsilon+m_{33}(\sin 2 \varepsilon)^{2}
\end{aligned}
$$

where the superscript $\mathrm{T}$ denotes transpose and $I_{\text {coelliptical }}$ is the intensity corresponding to the projection of $S_{\text {out }}$ on $S_{\mathrm{PSD}}$, i.e., for the coelliptical configuration.

Afterward, we operate in the same way, but now we project $S_{\text {out }}$ onto a polarization detector system configuring the orthogonal polarization, $S_{\mathrm{PSD}}^{\perp}$. To obtain the orthogonal polarization, it is sufficient to apply the following transformations to the polarization angles: $\varepsilon \rightarrow-\varepsilon$ and $\alpha \rightarrow \alpha+(\pi / 2)$. Under this scenario, the intensity resulting from the projection of $S_{\text {out }}$ on $S_{\mathrm{PSD}}^{\perp}$ (i.e., cross-elliptical configuration) becomes 


$$
\begin{aligned}
I_{\text {cross-elliptical }}= & \left(S_{\mathrm{PSD}}^{\perp, T}\right) \cdot S_{\text {out }} \\
= & (1-\cos 2 \varepsilon \cos 2 \alpha-\cos 2 \varepsilon \sin 2 \alpha-\sin 2 \varepsilon) \cdot S_{\text {out }} \\
= & m_{00}+m_{01} \cos 2 \varepsilon \cos 2 \alpha+m_{02} \cos 2 \varepsilon \sin 2 \alpha+m_{03} \sin 2 \varepsilon-m_{10} \cos 2 \varepsilon \cos 2 \alpha-m_{11}(\cos 2 \varepsilon \cos 2 \alpha)^{2} \\
& -m_{12}(\cos 2 \varepsilon)^{2} \cos 2 \alpha \sin 2 \alpha-m_{13} \cos 2 \varepsilon \cos 2 \alpha \sin 2 \varepsilon-m_{20} \cos 2 \varepsilon \sin 2 \alpha-m_{21}(\cos 2 \varepsilon)^{2} \cos 2 \alpha \sin 2 \alpha \\
& -m_{22}(\cos 2 \varepsilon \sin 2 \alpha)^{2}-m_{23} \cos 2 \varepsilon \sin 2 \alpha \sin 2 \varepsilon-m_{30} \sin 2 \varepsilon-m_{31} \cos 2 \varepsilon \cos 2 \alpha \sin 2 \varepsilon \\
& -m_{32} \cos 2 \varepsilon \sin 2 \alpha \sin 2 \varepsilon-m_{33}(\sin 2 \varepsilon)^{2}
\end{aligned}
$$

Finally, we perform the difference between Eqs. (5) and (6), leading to

$$
\begin{aligned}
I_{\text {co-elliptical }}-I_{\text {cross-elliptical }}= & 2 \cos 2 \varepsilon\left[m_{10} \cos 2 \alpha+m_{20} \sin 2 \alpha\right]+2(\cos 2 \varepsilon)^{2}\left[m_{11}(\cos 2 \alpha)^{2}+m_{22}(\sin 2 \alpha)^{2}\right. \\
& \left.+\cos 2 \alpha \sin 2 \alpha\left(m_{12}+m_{21}\right)\right]+2 \cos 2 \varepsilon \sin 2 \varepsilon\left[\cos 2 \alpha\left(m_{13}+m_{31}\right)+\sin 2 \alpha\left(m_{23}+m_{32}\right)\right] \\
& +2 \sin 2 \varepsilon\left[m_{30}+m_{33} \sin 2 \varepsilon\right] .
\end{aligned}
$$

Therefore, by measuring the experimental MM of a sample and by using Eqs. (5)-(7), it is possible to retrieve multiple PG configurations just by setting the proper values for $\alpha$ and $\varepsilon$. Note that once the MM is measured, multiple PG configurations are numerically derived without the necessity of experimentally implementing each one of them.

In the following, we derive the PG configurations described in Sec. 2.1 using the MM-based formalism. First, setting $\alpha=0 \mathrm{deg}$ and $\varepsilon=0 \mathrm{deg}$ (linear polarization oriented at the laboratory vertical) in Eqs. (5) and (6), they yield, respectively,

$$
I_{\text {co-linear }}=C 1=m_{00}+m_{01}+m_{10}+m_{11}
$$

and

$$
I_{\text {cross-linear }}=C 2=m_{00}+m_{01}-m_{10}-m_{11} \text {. }
$$

Note that Eqs. (8) and (9) are, respectively, equivalent to the configurations $\mathrm{C} 1$ and $\mathrm{C} 2$, as explained in Sec. 2.1. Accordingly, LConfig is as follows:

$$
I_{\text {co-linear }}-I_{\text {cross-linear }}=C 1-C 2=2\left[m_{10}+m_{11}\right] \text {. }
$$

Next, we select PG configurations based on elliptical polarizations with arbitrary ellipticity, $\varepsilon$, and the corresponding major axis parallel to one of the laboratory frame axis represented by $\alpha=0$ deg in Eqs. (5) and (6). Thus, they are reduced, respectively, to

$$
\begin{aligned}
I_{\text {co-elliptical }}= & C 3 \\
= & m_{00}+\cos 2 \varepsilon\left[m_{01}+m_{10}\right]+\sin 2 \varepsilon\left[m_{03}+m_{30}\right] \\
& +\cos 2 \varepsilon \sin 2 \varepsilon\left[m_{13}+m_{31}\right] \\
& +m_{11}(\cos 2 \varepsilon)^{2}+m_{33}(\sin 2 \varepsilon)^{2}
\end{aligned}
$$

and

$$
\begin{aligned}
I_{\text {cross-elliptical }}= & C 4 \\
= & m_{00}+\cos 2 \varepsilon\left[m_{01}-m_{10}\right]+\sin 2 \varepsilon\left[m_{03}-m_{30}\right] \\
& -\cos 2 \varepsilon \sin 2 \varepsilon\left[m_{13}+m_{31}\right]-m_{11}(\cos 2 \varepsilon)^{2} \\
& -m_{33}(\sin 2 \varepsilon)^{2}
\end{aligned}
$$

Note that Eqs. (11) and (12) are equivalent to the EPG configurations $\mathrm{C} 3$ and $\mathrm{C} 4$, respectively, both explained in Sec. 2.1. Accordingly, different ellipticities can be set by properly selecting the angle $\varepsilon$. Logically, for $\varepsilon=0$, we end up in the linear case, thus, Eqs. (11) and (12) reduce to Eqs. (8) and (9), respectively.

The EConfig described in Sec. 2.1 can be obtained by subtracting these two relations as follows:

$$
\begin{aligned}
& I_{\text {co-elliptical }}-I_{\text {cross-elliptical }}=C 3-C 4 \\
& =2 \cos 2 \varepsilon\left[m_{10}+m_{11} \cos 2 \varepsilon\right] \\
& \quad+2 \cos 2 \varepsilon \sin 2 \varepsilon\left[\left(m_{13}+m_{31}\right)\right] \\
& \quad+2 \sin 2 \varepsilon\left[m_{30}+m_{33} \sin 2 \varepsilon\right]
\end{aligned}
$$

Note that we can select an arbitrary EConfig simply by changing the $\varepsilon$ parameter in Eq. (13).

Finally, from the above-described formulation, we can also obtain the function $f$ defined in Eq. (1) and derived in Ref. 19. To avoid the requirement of performing extra experimental measurements for the determination of the parameter $\beta$ in Eq. (1), we set $\alpha=45 \mathrm{deg}$. By using this configuration, the amount of light projected to the $p$-polarized and $s$-polarized components is always the same, independent of the chosen ellipticity. Thus, the factor $\beta$ equals to 1 in such a case. Accordingly, Eq. (7) becomes

$$
\begin{aligned}
I(\alpha= & 45 \mathrm{deg})_{\text {co-elliptical }}-I(\alpha=45 \mathrm{deg})_{\text {cross-elliptical }} \\
= & 2 \cos 2 \varepsilon\left[m_{20}+m_{22} \cos 2 \varepsilon\right]+2 \cos 2 \varepsilon \sin 2 \varepsilon\left[m_{23}+m_{32}\right] \\
& +2 \sin 2 \varepsilon\left[m_{30}+m_{33} \sin 2 \varepsilon\right] .
\end{aligned}
$$

Moreover, if $\varepsilon=0 \mathrm{deg}$ in Eq. (14), it reduces to the linear case:

$$
\begin{aligned}
& I(\alpha=45 \mathrm{deg} ; \varepsilon=0 \mathrm{deg})_{\text {co-elliptical }} \\
& \quad-I(\alpha=45 \mathrm{deg} ; \varepsilon=0 \mathrm{deg})_{\text {cross-elliptical }}=2\left[m_{20}+m_{22}\right]
\end{aligned}
$$

Finally, adding Eqs. (14) and (15) leads to

$$
\begin{aligned}
f_{\mathrm{MM}}= & \left.(C 3-C 4)\right|_{\alpha=45}+\left.(C 1-C 2)\right|_{\alpha=45} \\
= & 2\left[m_{20}+m_{22}\right]+2 \cos 2 \varepsilon\left[m_{20}+m_{22} \cos 2 \varepsilon\right] \\
& +2 \cos 2 \varepsilon \sin 2 \varepsilon\left[m_{23}+m_{32}\right] \\
& +2 \sin 2 \varepsilon\left[m_{30}+m_{33} \sin 2 \varepsilon\right]
\end{aligned}
$$


which provides the function $f$ as a function of the MM coefficients.

Note that, in practice, many samples present spatial dependence of their polarimetric properties as birefringent samples that possess a principal direction. In such cases, larger image contrast may be obtained by illuminating the sample with a different azimuth angle. If this is the case, the parameter $\beta$ should be experimentally determined, as described in Ref. 19.

\section{Imaging Mueller Polarimeter and Sample Description}

In this section, we describe the optical arrangement that is used to measure the MM of samples (Sec. 3.1), and we describe the different samples we study in the forthcoming experimental section (Sec. 3.2).

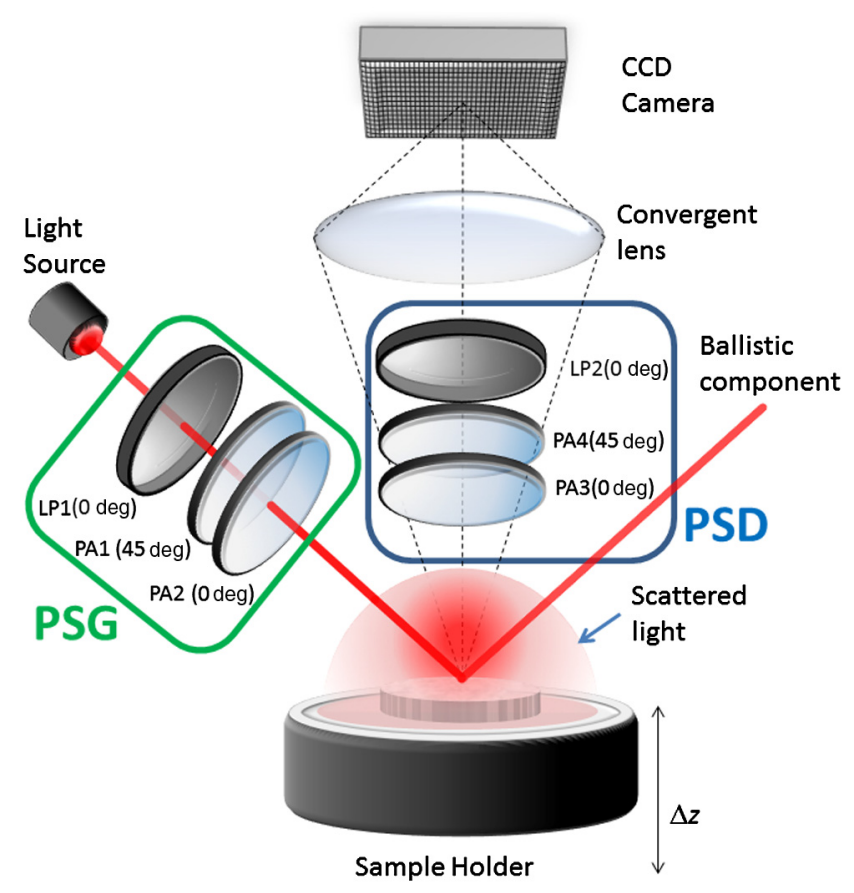

Fig. 1 Optical scheme of the used Mueller polarimeter. Video 1 shows the PG-based function $f_{\mathrm{MM}}$ calculated from the MM of the sample A. Different frames are related to different ellipticity values. (Video 1, MOV, 725 KB [URL: http://dx.doi.org/10.1117/1.JBO.22.5 $.056004 .1])$.

\subsection{Optical Scheme: Imaging Mueller Polarimeter}

The optical set-up used to measure MMs of different samples is a complete imaging MM polarimeter ${ }^{36}$ based on parallel aligned liquid crystal (PA-LC) retarders. The set-up, sketched in Fig. 1, is analogous to that described in Ref. 37 but adapted for imaging polarimetry.

First, a light source illuminates a polarization state generator (PSG, marked with a green rectangle in Fig. 1), which includes a linear polarizer at $0 \mathrm{deg}$ to the laboratory vertical reference axis, a first PA-LC panel (PA1), oriented at $45 \mathrm{deg}$ to the laboratory vertical, and a second PA-LC panel oriented at 0 deg to the laboratory vertical (PA2). As discussed in Ref. 37, any fully polarized state of polarization can be generated with this system by adjusting the retardances of the two PA-LC panels. Then, the input light with controlled polarization illuminates a sample holder, where the sample is set, with an incident angle of $\sim 60$ deg. The sample holder can be mechanically displaced in the $z$ direction for imaging purposes. Then, the light scattered by the sample is measured with a polarization state detector (PSD, marked with a blue rectangle in Fig. 1), which is constructed with the same optical elements as those in the PSG but with inverse order. Finally, a convergent lens images the sample on a CCD camera with a certain magnification.

Under this scenario, by properly generating a basis of known input polarizations with the PSG and measuring the corresponding Stokes vector of the scattered light with the Stokes polarimeter (PSD system), we construct a linear system [see Eq. (2)] from which the experimental MM of the sample can be obtained by applying an inversion method, as discussed in Refs. 21 and 38.

All experimental results shown and analyzed in a forthcoming section (Sec. 4) were obtained by experimentally implementing the optical scheme sketched in Fig. 1. The experimental set-up is shown in Fig. 2(a). As a light source, we used a fourwavelength high-power LED source (operated by DC4104 drivers distributed by Thorlabs). In particular, the color channels used in this work for conducting the different experimental measurements were the red channel [with a central wavelength of $625 \mathrm{~nm}$ and a spectral bandwidth (FWHM) of $18 \mathrm{~nm}$ ] and the blue channel (central wavelength of $470 \mathrm{~nm}$ and a FWHM of $10 \mathrm{~nm}$ ), with maximum output powers of 240 and $250 \mathrm{~mW}$, respectively. To achieve a FWHM of $10 \mathrm{~nm}$ with the blue channel, a dielectric bandwidth filter (Thorlabs) was used. The linear polarizer in the PSG (LP1) is a Glan-Thompson prism-based polarizer (by CASIX). The linear polarizer at the PSD (LP2) is a dichroic sheet polarizer (by Meadowlark Optics). For the

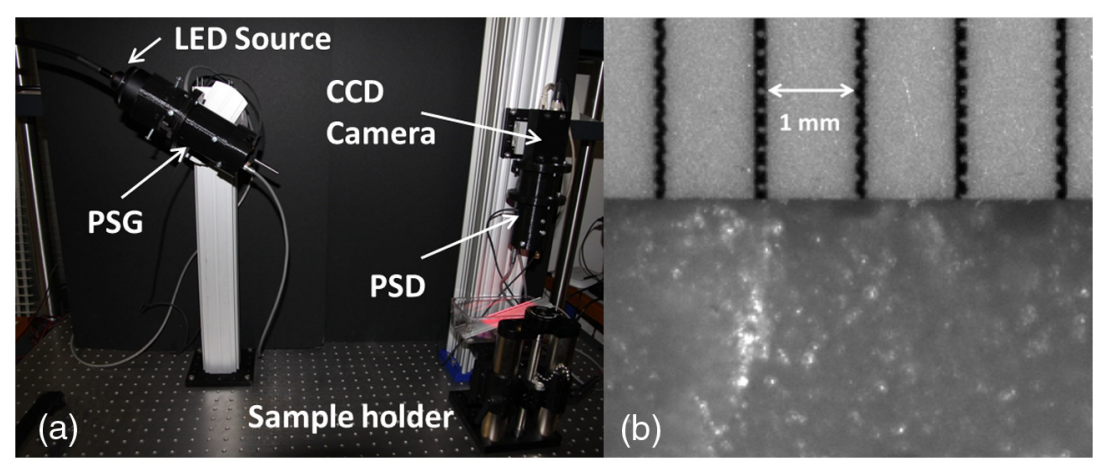

Fig. 2 (a) Picture of the experimental system; (b) image of a ruler placed on a slice of pork, for image resolution calculation. Video 2 shows the PG-based function $f_{\text {MM }}$ calculated from the MM of the sample B. (Video 2, MOV, 603 KB [URL: http://dx.doi.org/10.1117/1.JBO.22.5.056004.2]). 
liquid crystal panels in the PSG and PSA, we used four PA liquid crystal variable retarders with temperature control (LVR200-400-700-1LTSC by Meadowlark Optics). Finally, a microscope objective images the selected sample RoI on a CCD camera. The convergent lens in Fig. 1 was a TECHSPEC ${ }^{\circledR}$ high resolution objective, distributed by Edmund Optics, with a focal length of $35 \mathrm{~mm}$. The camera is an Allied Vision Manta G-504B. It is a 5 Megapixel GigE Vision camera with the Sony ICX655 CCD sensor, with a $2452(\mathrm{H}) \times 2056(\mathrm{~V})$ resolution, and a cell size of $3.45 \mu \mathrm{m} \times 3.45 \mu \mathrm{m}$. As shown in Fig. 2(a), the PSG and PSD systems were assembled in two black holders made with a 3-D printer. These holders were designed to configure a robust and feasible full-Mueller imaging polarimeter in which the optical elements were protected from the environment (dust, misalignments, stray light, scratches, etc.).

The resolution selected for the conducted experimental measurements is described in the following. In Fig. 2(b), we picture a ruler placed on a thick slice of pork tissue. The distance between different lines is $1 \mathrm{~mm}$. This distance corresponds to 52 pixels of the recorded image, leading to a spatial resolution of $\sim 19 \mu \mathrm{m}$. If more resolution is desired, the sample holder can be brought closer to the objective by performing an axial displacement $\Delta z$, as indicated in Fig. 1.

\subsection{Sample Description: Phantom Experiment and Ex-Vivo Measurement}

To prove that the above discussed PG configurations can be obtained from Mueller measurements, we have performed two different experiments.

First, we have reproduced the phantom experiment described in Ref. 19. Let us call it sample A. In particular, a metallic ruler is placed obliquely on a plastic tank $(15.5 \mathrm{~cm} \times 7.5 \mathrm{~cm} \times 5 \mathrm{~cm})$. Then the tank is filled with intralipid (20\%, Sigma-Aldrich, France) diluted in water [see Fig. 3(a)]. Intralipid is a lipid emulsion currently used for human intravenous use. In particular, intralipid is an emulsion of soy bean oil, egg phospholipids, and glycerin, and is available in $10 \%, 20 \%$, and $30 \%$ concentrations. In the current work, this intralipid is diluted in water to a concentration of $0.1 \%$, and the dilution is used to mimic the interaction of light with scattering tissues. For this concentration, the reduced scattering coefficients $\mu_{\mathrm{s}}^{\prime}$ for the red and blue channels are estimated to be of $\left.\mu_{\mathrm{s}}^{\prime}\right|_{625 \mathrm{~nm}}=0.63 \mathrm{~cm}^{-1}$ and $\left.\mu_{\mathrm{s}}^{\prime}\right|_{470 \mathrm{~nm}}=0.46 \mathrm{~cm}^{-1}$. According to Ref. 39, the corresponding anisotropy factors $g$ of the intralipid are 0.73 and 0.83 for the 625 and $470 \mathrm{~nm}$, respectively. The corresponding mean-free paths (MFPs) are $\left.\mathrm{MFP}\right|_{625 \mathrm{~nm}}=4.3 \mathrm{~mm}$ and MFP $\left.\right|_{625 \mathrm{~nm}}=3.7 \mathrm{~mm}$. According to the experimental results observed and described in a forthcoming section, with this configuration, we can perform macroscopic measurements with a sample penetration depth at the millimeter scale.

The second experiment is performed on an ex-vivo tissue (let us call it sample B). In particular, we have studied a region of interest (RoI) of a chicken neck, where different biological structures, such as muscles and nerves, are observed [see red square in Fig. 3(b)]. We want to emphasize that the region selected for measurements is fairly flat, avoiding possible image quality losses related to defocused planes.

Concerning the stability of the optical properties of the intralipid, a complementary experience, not discussed in this work, showed that the structure of an intralipid emulsion left still over time can evolve because spontaneous phase segregation (lipid/water) takes place. The process of phase segregation is
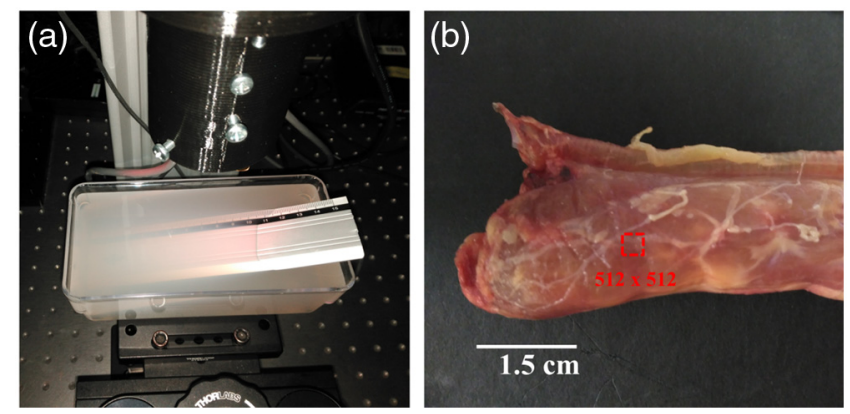

Fig. 3 (a) Tank filled with intralipid diluted in water and the metallic ruler (sample A). The sample is located in a sample holder to be measured with the polarimeter. The scattering effect is clearly observed. (b) Chicken neck for the ex-vivo experiment. The red square shows the measured Rol. Video 3 shows the PG-based function $f_{\mathrm{MM}}$ calculated from the equivalent diattenuator matrix (Di) for the sample B. (Video 3, MOV, 741 KB [URL: http://dx.doi.org/10.1117/1.JBO.22.5 .056004.3]).

very slow with a characteristic time of a few hours. Since the experiences reported here were performed in a time scale of seconds, we can, therefore, assume that the polarimetric properties of the samples remained constant throughout the measurement time.

\section{Experimental Results}

In this section, we provide the equivalence between the PG configurations and those deduced from MM (Sec. 4.1). In addition, the potential of using MM to perform PG-based analysis is highlighted in Sec. 4.2, as MM channels provide extra polarimetric information that can be combined with PG techniques.

To this aim, we show and discuss experimental data obtained from measurements on the samples described in Sec. 3.2 (see Fig. 3). In particular, different measurements of the samples were performed under different PG configurations. For comparison with formulation given in Sec. 2.2, the corresponding MMs of the samples were also measured. The two sets of measurements (i.e., PG configurations and MM measurements) were obtained by using the experimental set-up described in Sec. 3.1.

\subsection{Polarization Gating Configurations Derived from Mueller Measurements}

In this subsection, we provide the equivalence between different PG configurations (Sec. 2.1) and those obtained from MM measurements (Sec. 2.2). To this aim, we have used the sample A described in Sec. 3.2. All the images provided in this subsection present a resolution of $1024 \times 512$ pixels and are obtained by illuminating the samples with the red channel $(625 \mathrm{~nm})$.

First, the MM of the sample was experimentally obtained by using the Mueller polarimeter sketched in Fig. 1. To this aim, we used a basis of six states of polarization both for illumination and detection: linear polarizations oriented at 0, 45, 90, and $135 \mathrm{deg}$ to the laboratory vertical and two circular polarizations (right-handed and left-handed polarizations). The selected polarization basis stated above is commonly used due to its simplicity and because it leads to the minimum possible condition number (mathematical indicator that can be used to estimate the quality of a PSG-PSD system in terms of noise amplification) for polarimetric systems (Condition Number $=1.73$ ).

We want to note that some authors ${ }^{40,41}$ have proved that the intensity contrast of a particular sample can be enhanced by 
selecting a direct gating configuration, where the PSG-PSD polarizations are optimized. To make this selection of the optimal PSG-PSD channels, we need to know some a priori polarimetric information of the sample or measure its MM. In the latter case, from the measured MM, by applying an optimization method, one can find the PSG-PSD channels that maximize the image contrast. Note that the MM-based approach we propose not only can be combined with PG configurations but could also be used to perform the optimizations proposed in Refs. 40 and 41.

From the measured MM, different PG configurations were calculated according to the formulation described in Sec. 2.2. As an example, Fig. 4 shows the results obtained for some of the PG configurations described in Sec. 2.1, obtained by using the experimental MM coefficients: configurations C1 [Fig. 4(a)], C2 [Fig. 4(c)], C3 [Fig. 4(e)], C4 [Fig. 4(g)], C1-C2 [Fig. 4(i)], and $\mathrm{C} 3-\mathrm{C} 4$ [Fig. 4(k)].

For comparison, the same configurations were obtained by using standard PG procedures, i.e., by setting the proper PSGPSA configurations and recording the corresponding intensity images. Results are given in C1 [Fig. 4(b)], C2 [Fig. 4(d)], C3 [Fig. 4(f)], C4 [Fig. 4(h)], C1-C2 [Fig. 4(j)], and C3-C4 [Fig. 4(1)].

We see that the results obtained from MM or from standard PG techniques lead, in all cases, to equivalent information, because the final intensity images are very similar. To further quantify this similarity, Fig. 5 shows the absolute difference between the intensity images obtained from $\mathrm{MM}$ and from PG techniques for the C1 [Fig. 5(a)], C3 [Fig. 5(b)], C4 [Fig. 5(c)], and C3-C4 [Fig. 5(d)] cases.

For polarization configurations obtained from a single measurement, i.e., the $\mathrm{C} 1, \mathrm{C} 2, \mathrm{C} 3$, and $\mathrm{C} 4$ channels, the absolute error calculations were conducted as follows: (1) the $C_{i}(\mathrm{PG})$ image is obtained from PG measurements (the subscript $i$ is equal to 1, 2, 3, or 4 for the $\mathrm{C} 1, \mathrm{C} 2, \mathrm{C} 3$, and $\mathrm{C} 4$ cases, respectively); (2) the $C_{i}(\mathrm{MM})$ image is obtained from MM measurements; (3) $C_{i}(\mathrm{PG})$ and $C_{i}(\mathrm{MM})$ are normalized to 1 ; and (4) the absolute error between images $\left[C_{i}(\mathrm{PG})-C_{i}(\mathrm{MM})\right]$ is obtained pixel-to-pixel. (a)

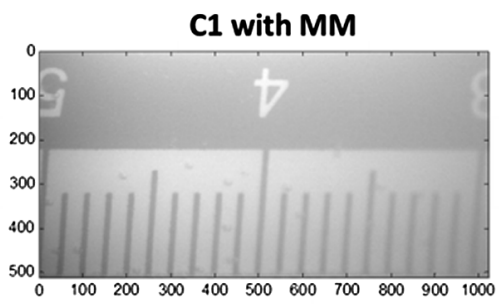

Pixels

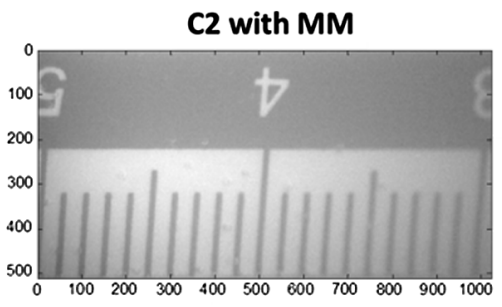

(c)

Pixels

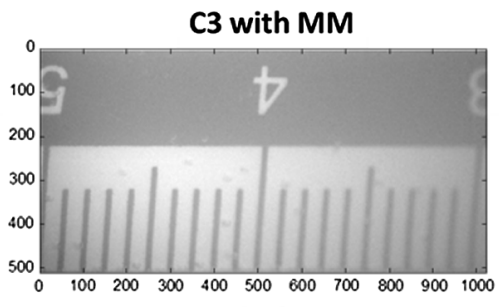

(e)

Pixels

C4 with MM

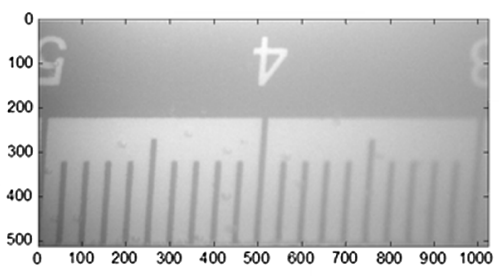

(g)

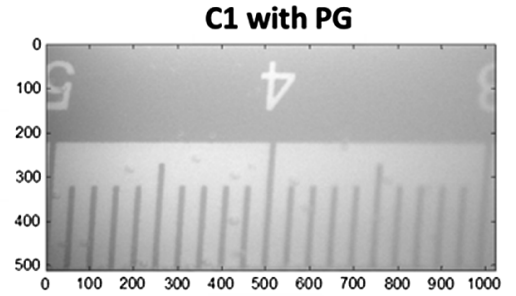

(b)

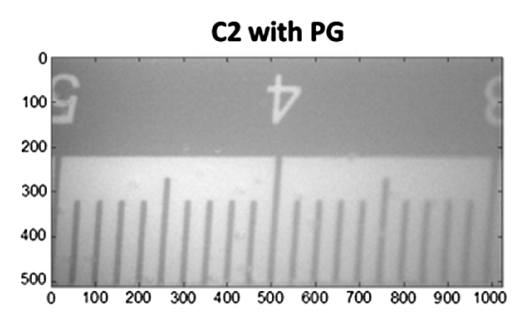

(d)

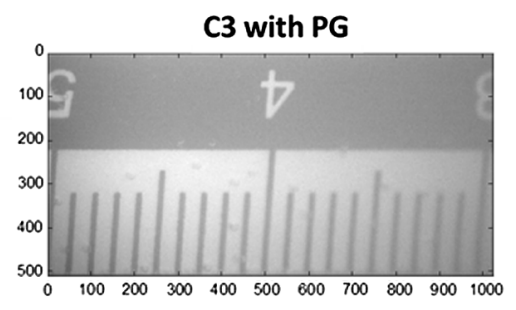

(f)

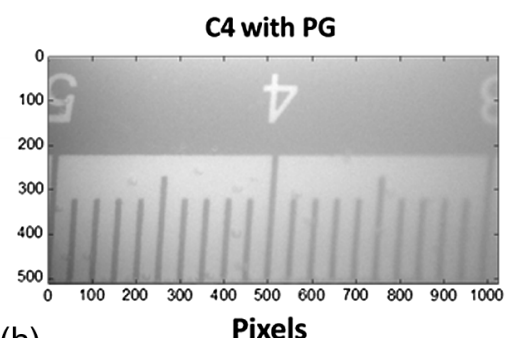

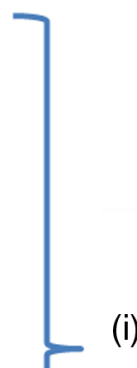

(i)
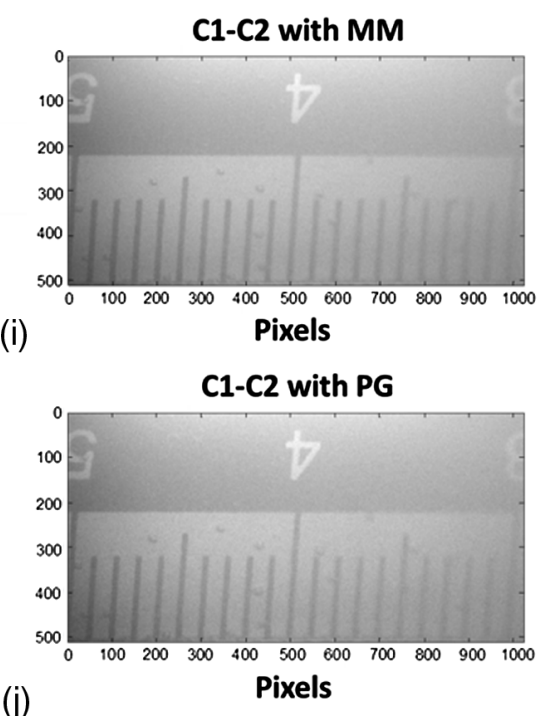

C3-C4 with MM

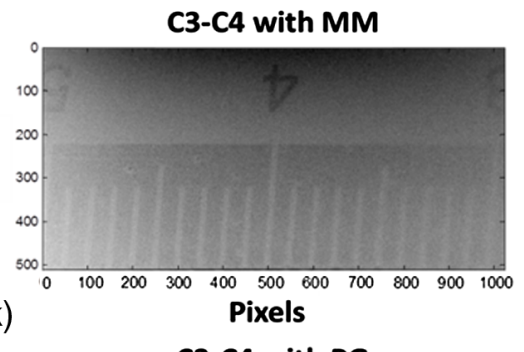

(k)
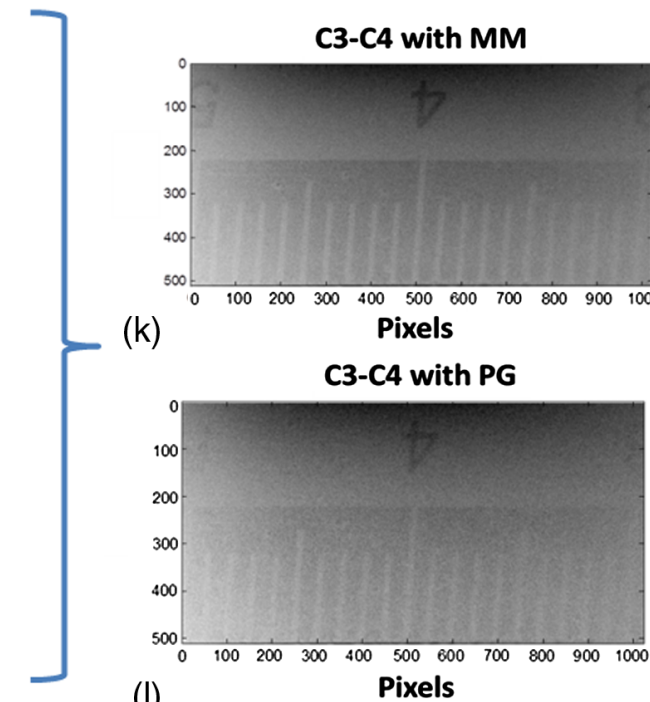

(I)

Fig. 4 Comparison between different PG configurations obtained from: MM coefficients (a, c, e, g, i, and $\mathrm{k}$ ); and from standard PG procedures (b, $\mathrm{d}, \mathrm{f}, \mathrm{h}, \mathrm{j}$, and $\mathrm{I}$ ). Video $4 \mathrm{PG}$-based function $f_{\mathrm{MM}}$ calculated from the equivalent depolarizer matrix (De) for the sample B. (Video 4, MOV, 974 KB [URL: http://dx.doi.org/10 $.1117 / 1 . J B O .22 .5 .056004 .4])$. 
For polarization configurations obtained from image processing, e.g., $\mathrm{C} 1-\mathrm{C} 2$ and $\mathrm{C} 3-\mathrm{C} 4$ channels, the absolute error calculations were conducted as follows: (1) the $C_{j}(\mathrm{PG})$ image is obtained from PG measurements (the subscript $j$ is equal to 1 or 3 for the $\mathrm{C} 1-\mathrm{C} 2$ or the $\mathrm{C} 3-\mathrm{C} 4$ cases, respectively); (2) the $C_{j}(\mathrm{MM})$ image is obtained from MM measurements; (3) the $C_{j+1}$ (PG) image is obtained from PG measurements; (4) the $C_{j+1}(\mathrm{MM})$ image is obtained from MM measurements; (5) the $C_{j}-C_{j+1}$ channels are obtained in each case: $A=$ $C_{j}(\mathrm{PG})-C_{j+1}(\mathrm{PG})$ and $B=C_{j}(\mathrm{MM})-C_{j+1}(\mathrm{MM})$; (6) the processed images $A$ and $B$ are normalized to 1 ; and (7) the absolute error between $A$ and $B$ is calculated as $A-B$.

In addition, the mean absolute error and standard deviation $(\sigma)$ values corresponding to all the experiments shown in Fig. 4 are also provided in Table 1.

Note that for direct PG channels, i.e., C1, C2, C3, and C4 configurations, the absolute differences [Figs. 5(a)-5(c)] and the mean absolute differences (Table 1) are always smaller than 0.05 . This leads to absolute differences smaller than $5 \%$ if taking into account the full images intensity range [0-1], and they can be mainly attributed to random noise at the intensity measurements. The case of PG channels obtained from image subtraction processing is also studied. We analyzed two different configurations: (i) the $\mathrm{C} 1-\mathrm{C} 2$ channel (i.e., " $\mathrm{C} 1$ $\mathrm{C} 2$ with $\mathrm{MM}$ "- "C1-C2 with PG") and (ii) the C3-C4 channel (i.e., "C3-C4 with MM"- "C3-C4 with PG"). The absolute errors between channels are always smaller than $0.1(10 \%)$ [e.g., Fig. 5(d)]. In addition, the mean absolute errors were 0.021 and 0.041 (Table 1), and thus smaller than $0.05(5 \%)$.

Note that the above-shown error values ensure the equivalence between standard PG configurations and PG configurations obtained from Mueller matrices. If required, these error values could be even more reduced by time-averaging different intensity images.

Finally, we want to highlight that by implementing PG configurations from MM measurements, we do not need to perform any new experiment for each extra PG configuration to be implemented. In fact, by using the mathematical formalism detailed in Sec. 2.2, a widespread number of PG configurations can be analytically obtained based on the MM coefficients.

As an example, we show the results obtained when implementing the function $f_{\mathrm{MM}}$, detailed in Eq. (16), from the MM coefficients measured for the phantom experiment (i.e., ruler submerged into a tank with intralipid diluted in water; see sample A description in Sec. 3.2). Note that the ellipticity angle $\varepsilon$ is

Table 1 Mean absolute difference and standard deviation $\sigma$ for different PG channels.

\begin{tabular}{lcc} 
Channel & Mean absolute difference & $\sigma$ \\
\hline C1 & 0.015 & 0.010 \\
C2 & 0.026 & 0.019 \\
C1-C2 & 0.021 & 0.016 \\
C3 & 0.020 & 0.014 \\
C4 & 0.012 & 0.009 \\
C3-C4 & 0.041 & 0.031 \\
\hline
\end{tabular}

(a)

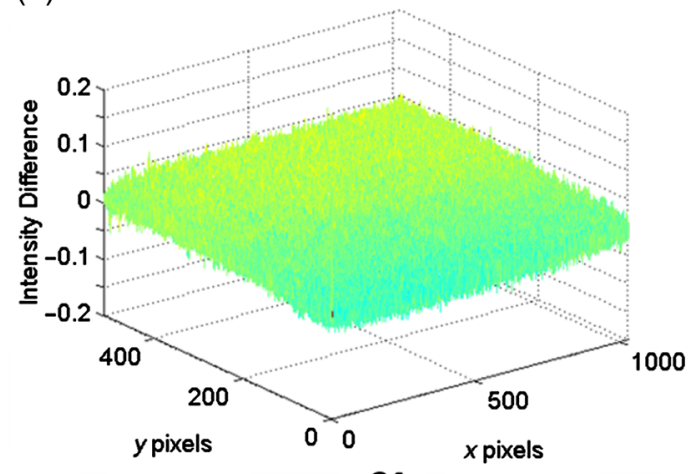

(c)

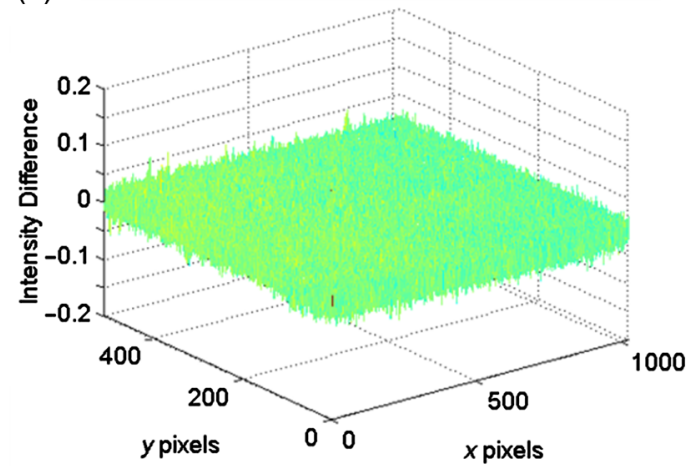

(b)

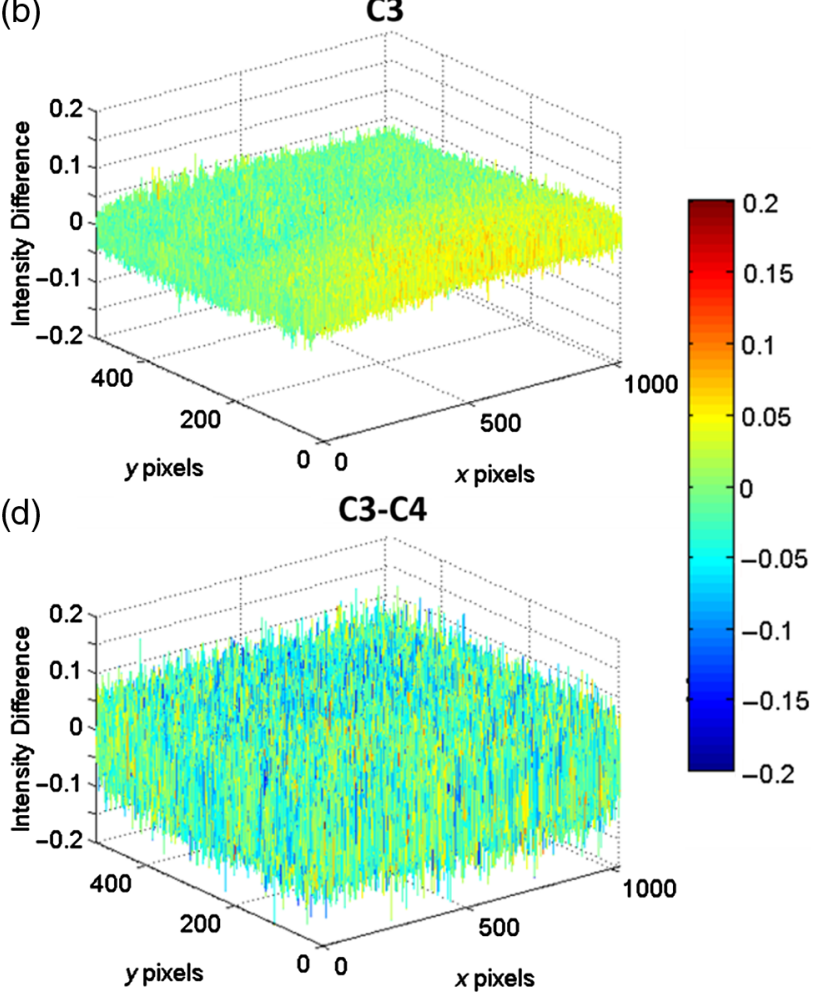

Fig. 5 Intensity absolute differences for the PG configurations: (a) C1, (b) C3, (c) C4; and (d) C3-C4. Video 5 shows PG-based function $f_{\mathrm{MM}}$ calculated from the equivalent retarder matrix (Ret) for the sample B. (Video 5, MOV, 1.03 MB [URL: http://dx.doi.org/10.1117/1.JBO.22.5.056004.5]). 
a control parameter in Eq. (16). Therefore, in contrast to traditional PG experiments, where each ellipticity value to be tested (i.e., ellipticity for the generated and analyzed polarizations) has to be experimentally implemented, we can readily obtain such information with an arbitrary $\varepsilon$ from measured MM coefficients [Eq. (16)]. This situation is highlighted in Video 1, where different frames show the sample A image obtained by processing the function $f_{\mathrm{MM}}$ as a function of the $\varepsilon$ value, from $\varepsilon=-45 \mathrm{deg}$ to $\varepsilon=+45 \mathrm{deg}$, with steps of $1 \mathrm{deg}$. Note that each video in this manuscript can be visualized with the corresponding link placed at the figure caption of the figure with the same number.

Images of Video 1 show the ruler submerged into the diluted intralipid and the different ruler numbers observed (i.e., 3-4-5) are related to different depths in the liquid [see Fig. 3(a)]. In particular, the number 5 is the number closer to the surface and the number 3 is the one more in depth. In agreement with other studies, ${ }^{17,19}$ we see that larger ellipticity values $(\varepsilon \sim \pm 45 \mathrm{deg})$ lead to a larger quantity of photons coming from deeper parts of the sample, as the number of scattering events taking place in a particular length depend on the polarization. This can be observed in Video 1 as clearer $(\varepsilon \sim+45 \mathrm{deg})$ or darker $(\varepsilon \sim 0 \mathrm{deg})$ image frames.

To quantify the different contrast provided by selecting different ellipticities in Eq. (16) for the phantom experiment (variations observed in Video 1), we have calculated the visibility $V=\frac{I_{\max }-I_{\min }}{I_{\max }+I_{\min }}$ for the two cross-sections shown in Fig. 6(a) (blue line for the four number and red line for the ruler tick mark), where $I_{\max }$ and $I_{\min }$ are the maximum and minimum values in the cross-section selected. We want to note that the best visibility values are obtained for an elliptical state of polarization ( $\sim 30 \mathrm{deg})$. In general, PG configurations based on linear and circular polarizations are commonly used for its simplicity to be experimentally implemented. Howerver, as shown in Fig. 6(b), these configurations will not always lead to the best contrast. If another ellipticiy needs to be tested, by using standard PG measurements, each new configuration has to be experimentally implemented, leading to a blind process. However, by using the method described in Sec. 2.2, such information can be analytically obtained, as shown in Fig. 6(b).

Note that Video 1 and data in Fig. 6 show the potential of rapidly implementing standard PG configurations from
MM measurements, as this method constitutes a more general approach.

\subsection{Polarization Gating and Mueller Processing Combination}

In this section, we show the interest of performing polarimetric analysis of biological samples using $\mathrm{MM}$ data because this method not only allows to set multiple PG configurations from analytical expressions (shown in Sec. 4.1), but also provides new information channels (Mueller matrices, matrix decompositions, differential matrices, etc). In addition, the study provided in this section also highlights the interest of combining MM analysis tools with PG configurations.

\subsubsection{Phantom experiment}

We first performed a modification on the sample A by also considering retardance. The intralipid-ruler based experiment discussed above tries to mimic the scattering response of biological tissues. However, in general, other polarimetric responses simultaneously occur when light interacts with biological tissues. For instance, most biological tissues contain collagen fibers, which are part of the extracellular matrix and provide structural and biochemical support to the surrounding cells. Depending on the collagen degree of mineralization, collagen tissues may be rigid (e.g., bone), flexible (e.g., tendon), or present a gradient from rigid to flexible (e.g., cartilage). Moreover, collagen fibers are birefringent materials that present different retardation values for different biological structures, ${ }^{42}$ such as those present in nerves, muscles, bones, ligaments, etc. Thus, image contrast between different tissues may be enhanced by considering polarimetric information. Some studies show that collagen fibers appear curly and anisotropic in normal stroma, ${ }^{43}$ but they appear more stretched during early cancer progression, and they tend to be aligned parallel to the tumor border. ${ }^{44}$ Therefore, retardation content is a valuable parameter for enhancing image contrast for biological and medical applications.

To take this fact into account and to include some retardance in the sample, the intralipid-ruler based experiment was repeated but by sticking two cellophane tape films on the ruler with different orientations, respectively [let us call it sample C; (a)

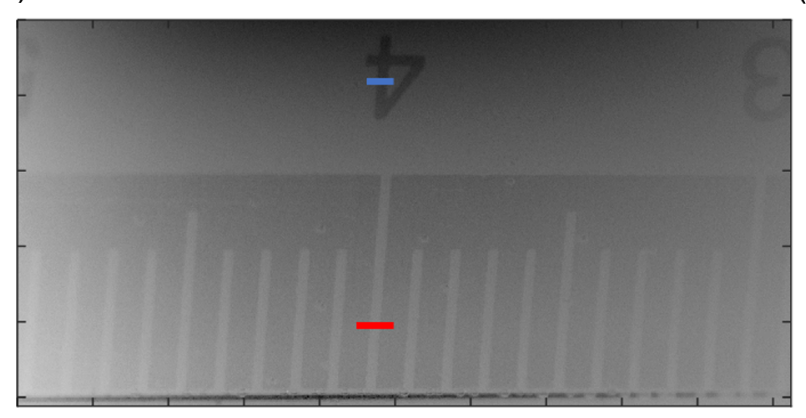

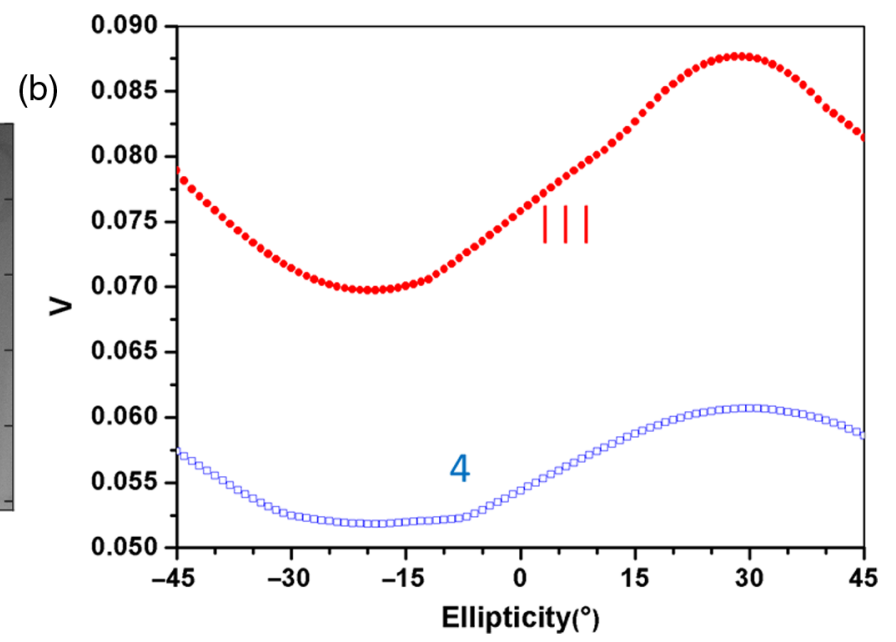

Fig. 6 (a) Cross-sections selected for the number 4 (blue line) and a ruler millimeter tick mark (red line). (b) Visibility (V) as a function of the ellipticity angle. 
(a)

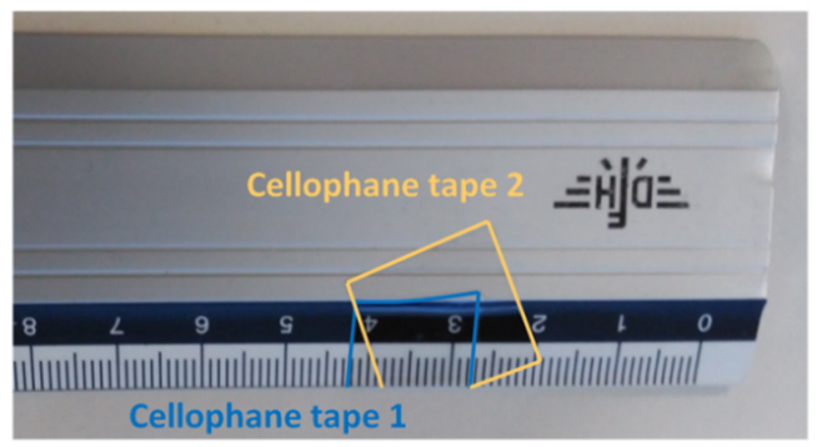

(c)

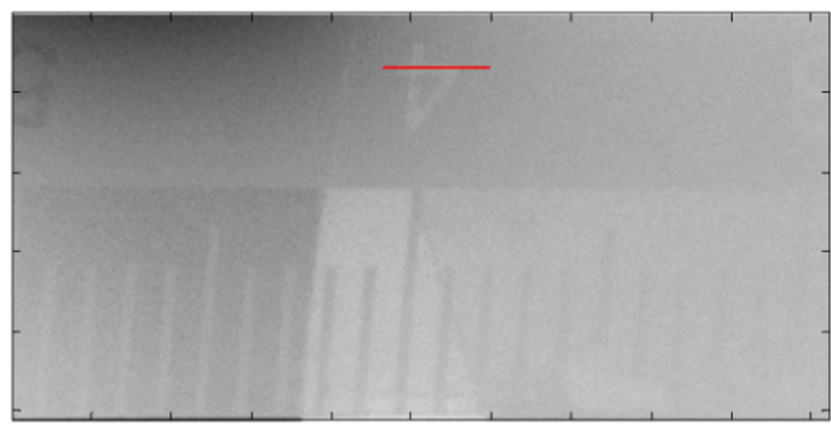

$625 \mathrm{~nm}$ (b)

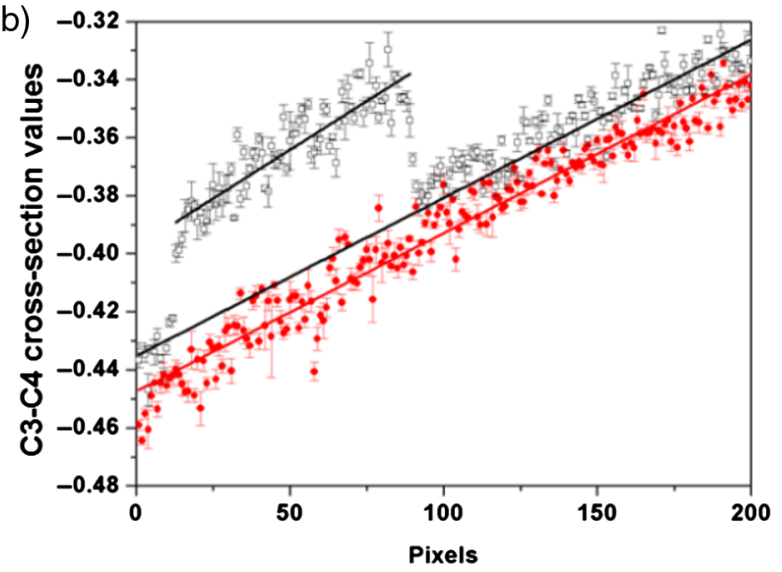

(d)

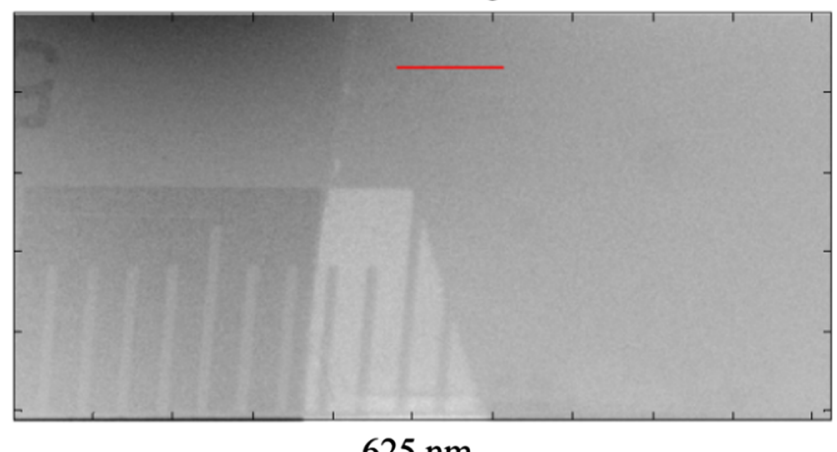

Fig. 7 (a) Ruler with stuck Scotch tapes (sample C); (b) cross-section values of the red line in panel (c) (black empty squares) and panel (d) (red circles); (c) C3-C4 PG image for right-handed circular light; and (d) C3-C4 PG image for left-handed circular light.

see Fig. 7(a)]. Note that the cellophane tape films act as linear retarders due to the stress applied on their constituent polymers during the fabrication process.

First, we have measured sample $\mathrm{C}$ by using traditional $\mathrm{PG}$ experimental techniques with the 625 -nm light channel. In particular, Fig. 7(c) shows the image obtained by performing the standard PG configuration labeled as C3-C4 in Sec. 2.1, by using right-handed circular polarization for illumination. By contrast, Fig. 7(d) shows the same PG configuration but this time for a left-handed circular illumination. We can see welldifferentiated zones with different intensity structures in sample $\mathrm{C}$ as a consequence of the polarization variations induced by the cellophane retardance. Note that these differences are not present in Figs. 4(k) and 4(1) (same PG configuration but applied on sample A). In addition, we observe that image contrast is significantly different by using right-handed [Fig. 7(c)] or left-handed [Fig. 7(d)] polarizations.

For example, the number 4 is not visible for the left-handed polarization, but it is clearly visible when using the right-handed polarization. This fact is highlighted in Fig. 7(b), where the cross-section corresponding to the red lines marked in Figs. 7(c) and 7(d) is shown. The cross-section values related to the red line in Fig. 7(c) (right-handed polarization case) go linearly to zero as we go inside the intralipid-water solution (see red circles). The linear tendency is shown by the calculated linear regression drawn as a continuous line (red line). In turn, the cross-section values for the red line in Fig. 7(d) (left-handed polarization case) have the same behaviour but show a sharp jump in the image contrast for the pixels related to the number
4 (see black squares). This situation occurs because elliptical states of polarization are modified in a different way depending on the retardation and orientation of the cellophane tape. Although some authors have pointed out the interest of using elliptical polarizations to test deeper structures in biological tissues, data shown in Fig. 7 also show the interest of optimizing the helicity of the selected polarization, because such a parameter can be important to enhance image contrast in biological tissues.

Afterward, we have calculated the MM of the sample $\mathrm{C}$ for $625 \mathrm{~nm}$. The corresponding 16 real images are shown in Fig. 8. We want to note that the image coefficients pictured in Fig. 8 are normalized in each case by the corresponding maximum coefficient value, thus maximizing the image contrast for each particular coefficient. However, this representation does not allow us to visualize the relative intensity differences between Mueller coefficients. Thus, a matrix showing the maximum intensity value for each MM element when being normalized by the maximum value of the MM00 element is also provided as a bar chart at the left part of Fig. 8. This information allows us to identify the relative intensity magnitude between channels.

Note that the MM by itself provides different polarimetric information (MM coefficients in Fig. 8) than those related to the standard PG configurations, thus, MM coefficients provide further information that can be used and combined to enhance the sample image contrast. ${ }^{45}$ In addition, the MM analysis allows performing a physical interpretation of the sample. ${ }^{21,22}$ In a rough approximation, the MM coefficients in the first row and column of the matrix can be linked with the diattenuation 


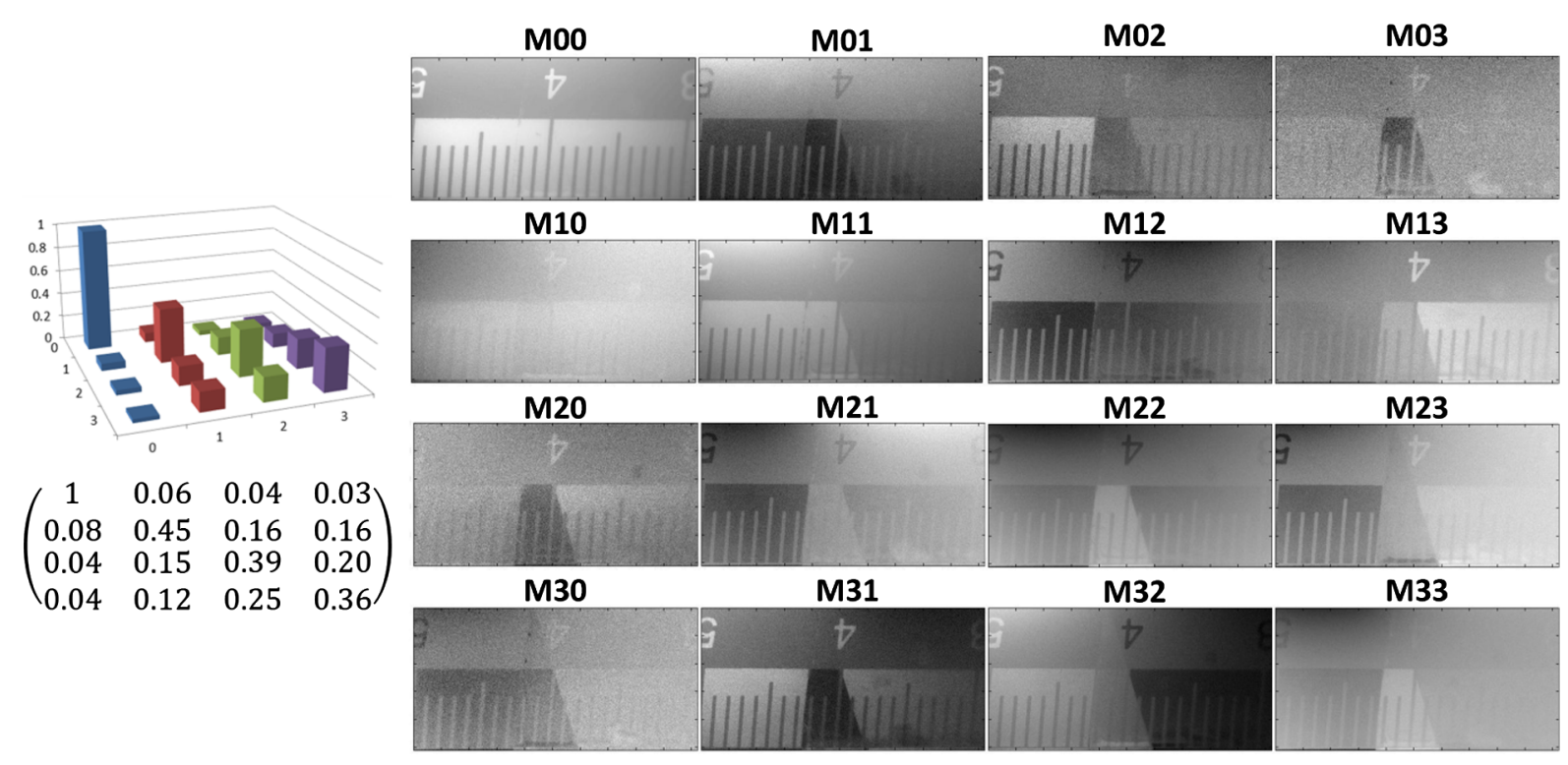

Fig. 8 Experimental MM of the sample C measured at $625 \mathrm{~nm}$.

and polarizing capabilities of the sample, respectively (in the case of the sample $\mathrm{C}$, mainly related to light reflections on the metallic surface of the ruler), the diagonal coefficients encode the depolarizing capability of the sample (for the sample C, mainly related with scattering events produced due to the fats present in the intralipid-water dissolution), and the bottomright $3 \times 3$ submatrix can be linked with the retardance introduced by the sample (in sample $\mathrm{C}$, due to the cellophane birefringence values).

In addition, the polarimetric content encoded on the MM of the sample can be further quantified by performing a physical model, where the properties of the sample are thought of as a combination of different pure polarimetric samples, typically, a diattenuator, a retarder, and a depolarizer. In other words, the sample can be further inspected by using well-known MM analytical tools, such as by applying MM decompositions. ${ }^{21,22,46}$

\subsubsection{Ex-vivo experiment}

Finally, to discuss a case which is closer to real experience than the phantom previously shown, the ex-vivo tissue labeled as sample B (see Sec. 3.2) was analyzed. To this aim, the MM of the sample was measured using the 470-nm channel. Some authors have provided the dependence of light penetration in biological tissues with the wavelength used. ${ }^{47}$ In the visible range, larger wavelengths enter deeper into the skin than shorter ones. Therefore, (a)

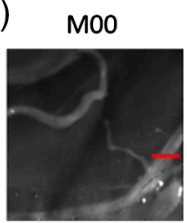

M10

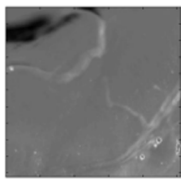

M20

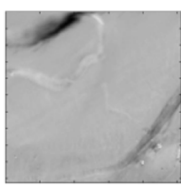

$\mathrm{M} 30$

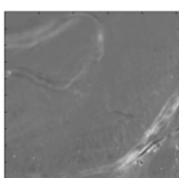

M01

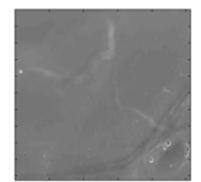

M11

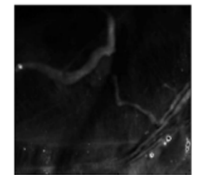

M21

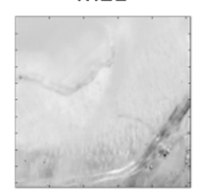

M31

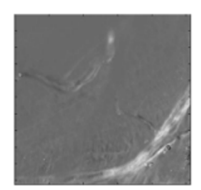

$\mathrm{MO2}$

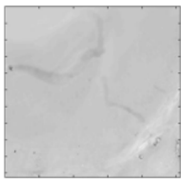

M12

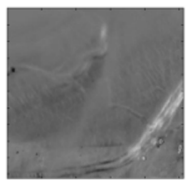

M22

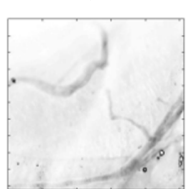

M32

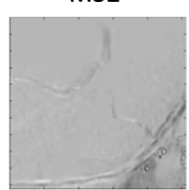

$\mathrm{MO3}$

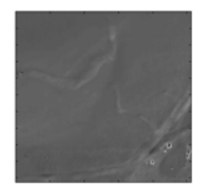

M13

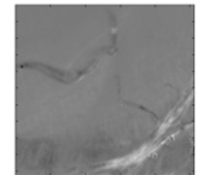

$\mathrm{M} 23$

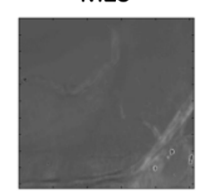

M33

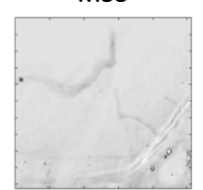

(b)
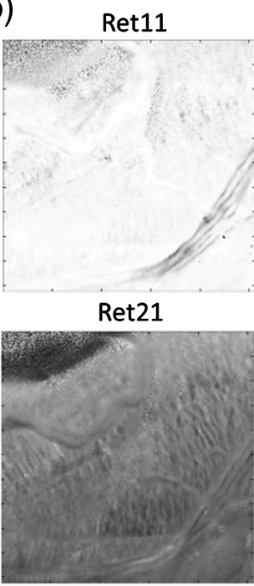

Ret31

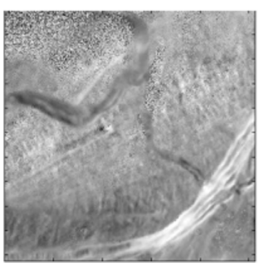

Ret12
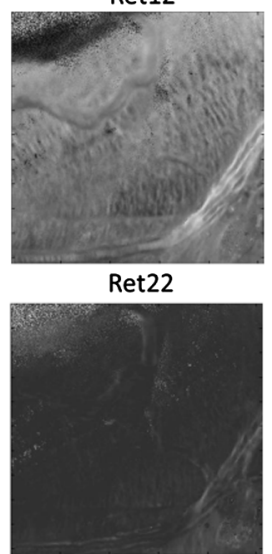

Ret32

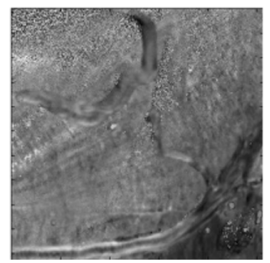

Ret13

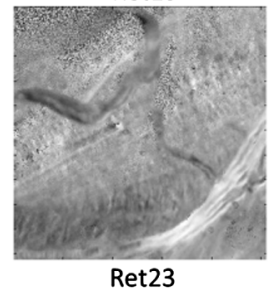

Ret23

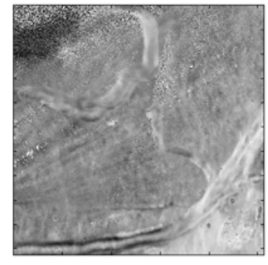

Ret33

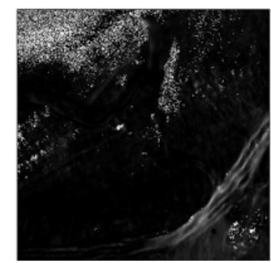

Fig. 9 (a) Experimental MM of the sample B measured at $470 \mathrm{~nm}$ and (b) $3 \times 3$ Mueller submatrix of the equivalent linear retarder for the sample $B$. 
we have chosen the shorter available wavelength for illumination to mainly inspect biological structures at the sample surface.

The experimental MM for the sample B is shown in Fig. 9(a). As can be observed from the coefficient M00, which is related to the sample irradiance, the selected RoI [see Fig. 3(b)] includes different biological structures, such as muscles and nerves. It can be observed that different image coefficients lead to different contrast levels between the biological structures present in the sample [Fig. 9(a)].

In addition, to highlight the interest of using MM-based analytical tools, we have also applied the Lu-Chipman decomposition, ${ }^{22}$ which allows us to decompose the MM of a sample as a product of three basic MMs: the MM of a diattenuator (Di), the MM of a retarder (Ret), and the MM of a depolarizer (De). As an example, the MM of the retarder (Ret) for sample B is also provided in Fig. 9(b). For a better visualization of the Ret matrix, the first row and column coefficients are removed in Fig. 9(b) because they are always zero in linear retarders. Thus, significant polarimetric content in the Ret matrix is at the $3 \times 3$ submatrix shown in Fig. 9(b).

Note that the matrix Ret only provides the retardance information of the sample (other polarimetric content has been already extracted by means of the matrix decomposition), thus, the image contrast observed in the Ret matrix channels is mainly due to retardance features of the sample. In other words, contrast obtained from the retarder matrix of biological tissues is related to retardance created by different sample structures, for instance, due to the different collagen fibers density, mineralization degree, or orientation at the different biological structures. For the sample B particular case [Fig. 9(b)], we see that the retardance content of the sample provides a noticeable contrast of the nerve ramification along the muscle in the chicken neck, this being specially highlighted in the Ret13, Ret31, Ret23, and Ret 32 coefficients of the Ret matrix. In addition, the MMs of the depolarizer (De) and the diattenuator (Di) have been calculated as well, leading, in this case, to less contrast than the $\mathrm{M}$ and the Re matrices.

To quantify the above-stated discussion, in Fig. 10, we show the cross-sections related to the red line shown in Fig. 9 [see coefficient M00 in Fig. 9(a)]. In particular, we have calculated the visibility corresponding to the cross-line above-stated for

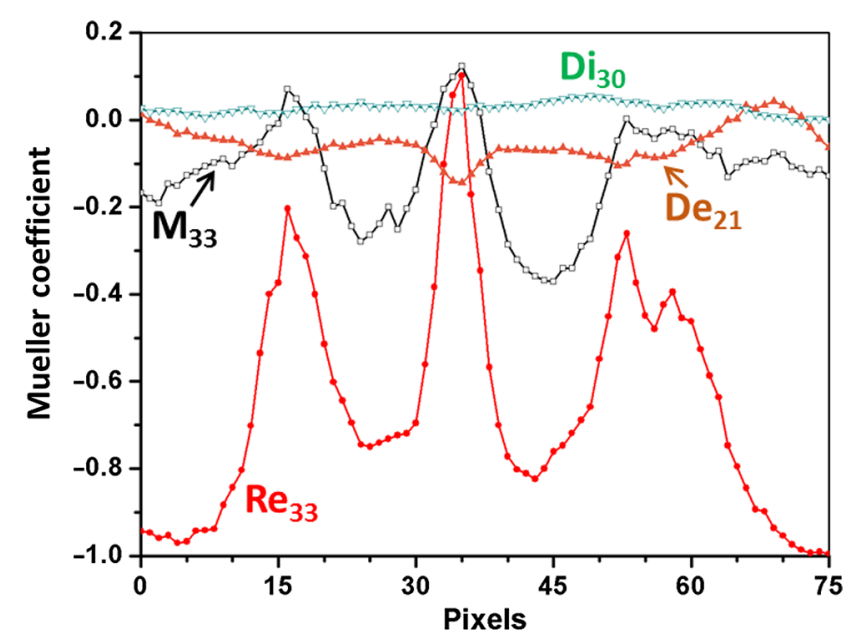

Fig. 10 Cross-sections [related to the line shown in Fig. 9(a)] corresponding to the coefficients providing the best visibility for the MM (black empty squares), the retarder (red circles), the depolarizer (brown triangles), and the diattenuator (green empty triangles). all the Mueller coefficients and for all the coefficients of the decomposed matrices (Di, De, and Ret cases), always for the same line of the sample. The largest visibility in each case has been obtained for the M33, Ret33, De21, and Di30 coefficients. The corresponding cross-sections are provided in Fig. 10.

In agreement with the qualitative discussion provided above, the best contrast for the chicken nerve [see red line in Fig. 9(a)] is obtained with the retarder matrix, where the separation between different nerve structures is highlighted by the different picks (see red circles in Fig. 10). Note that this sample detail is almost hidden in the diattenuation and the depolarization channels (green empty triangles and brown triangles, respectively, in Fig. 10).

At this point, we have shown how PG techniques based on MM measurements lead to a generalization of the standard PG methods (Secs. 2.2 and 4.1). In this subsection, we also highlighted the interest of using MM measurements for biological tissues inspection as they bring a whole new battery of analytical techniques. Last but not least, we want to discuss the interest of combining PG methods with MM analytical tools.

To this aim, we used sample B [chicken neck, see RoI in Fig. 3(b)] described in Sec. 3.2. In particular, the function $f_{\text {MM }}$ [Eq. (16) in Sec. 2.2; PG-based relation] was calculated from the $\mathrm{MM}$ coefficients of sample $\mathrm{B}$ [data pictured in Fig. 9(a)]. In addition, to exploit the MM capabilities, the $f_{\text {MM }}$ was calculated again but this time from the decomposed Mueller matrices, which were calculated according to the Lu-Chipman decomposition. ${ }^{22}$ Therefore, the $f_{\mathrm{MM}}$ function was obtained for the diattenuator (Di), the retarder (Ret), and the depolarizer (De) Mueller matrices. As $f_{\mathrm{MM}}$ is an analytical expression, in all the cases, it was calculated for different ellipticity values, from -45 to $+45 \mathrm{deg}$ with steps of $1 \mathrm{deg}$. Thus, results are provided in video format. In particular, Video 2 provides the $f_{\mathrm{MM}}$ function calculated from the MM as a function of the ellipticity value selected. Moreover, the same function but calculated from the $\mathrm{Di}$, De, and Ret matrices is given in Video 3, Video 4, and Video 5, respectively.

On the one hand, when analyzing the data obtained from the MM coefficients (Video 2), we observe certain contrast between the biological tissues present in the sample, mainly nerves and muscle tissues. Moreover, the biological structures visualization strongly depends on the ellipticity of the PG channel selected. For instance, in Video 2, we see that the nervous tissue placed at the image bottom is better visualized by using larger ellipticities ( $\varepsilon \sim \pm 45 \mathrm{deg}$ ), showing more clearly its ramified structure. On the contrary, surface muscle is better contrasted by using linear polarizations $(\varepsilon \sim 0 \mathrm{deg})$.

On the other hand, better sample interpretation can be achieved by using the $f_{\mathrm{MM}}$ function derived from decomposed Mueller matrices. First, Video 3 provides the $f_{\text {MM }}$ function calculated from the diattenuator matrix coefficients. As can be observed, we obtain a very poor image contrast between tissues, indicating that the analyzed structures do not present significant dichroism values (at least at the superficial layers), and polarimetric features of sample B are mainly related to birefringence and depolarizing processes. Next, the $f_{\mathrm{MM}}$ function calculated from the depolarizer matrix coefficients provides a noticeable image contrast between nerves and muscles (Video 4), indicating that each particular tissue scatters light in a different way. In addition, image contrast varies with the selected elliptical channel, ellipticities close to $\varepsilon \sim-45 \mathrm{deg}$ being those providing the best contrast between nerves and muscle. By contrast, the fibrillary nature of muscle tissue is 
better resolved by using linear channels ( $\varepsilon< \pm 10 \mathrm{deg}$ ). Finally, the function $f_{\mathrm{MM}}$ calculated from the retarder matrix Ret also provides different polarimetric information (Video 5). By using this polarimetric information, muscle-nerve contrast is reduced when compared with data in Video 4. In addition, muscle tissue seems to be quite uniform through the whole muscle surface, leading to a uniform black image (the best muscle contrast is obtained for linear channels). Nevertheless, nerve contrast itself is significant. In fact, $f_{\mathrm{MM}}$ function based on the retarder data reveals a ramified structure for the nerve (in the lower part of the images), where different nerve details are discovered by using different ellipticity channels.

We want to emphasize that this particular example could be further investigated by using other analytical expressions deducted from MM coefficients, as those provided in Ref. 45, or other relevant processing techniques.

\section{Conclusions}

We presented an experimental method based on the calculation of the MM of samples suitable for the calculation of standard PG techniques. We proved that this method is not only equivalent to standard PG measurements, but also can be used to build general analytical expressions from which a set of $\mathrm{PG}$ configurations can be simultaneously obtained.

We also proved that when conducting PG measurements to inspect biological tissues, it is not only important to optimize the ellipticity of the input and analyzing states of polarization, as previously highlighted by some authors, but also to optimize the helicity of the elliptical state of polarization. This situation occurs because when polarized light interacts with complex structures, right-handed and left-handed polarizations are modified in a different way due to different media polarimetric characteristics, for instance, the retardance introduced by the media, the orientation of the extraordinary axis of the anisotropic media, etc.

Finally, we have also highlighted the interest of not only performing PG based on MM measurements but also to take advantage of all those analytical tools related to the Mueller formalism. By doing this, extra polarimetric channels and analysis techniques are available. In addition, they can be combined with standard PG methods to obtain further information.

The experimental method proposed here was tested on different samples as different phantom experiments and ex-vivo biological tissues, thereby providing the suitability of the method to be applied for the polarimetric analysis of samples.

\section{Disclosures}

No conflicts of interest, financial or otherwise, are declared by the authors.

\section{Acknowledgments}

We acknowledge the financial support of Spanish MINECO (FIS2015-66328-C3-1-R, FIS2015-66328-C3-3-R, and Fondos FEDER); Catalan Government (SGR 2014-1639).

\section{References}

1. T. Novikova et al., "Polarized light for biomedical applications," J. Biomed. Opt. 21(7), 071001 (2016).

2. A. G. Ushenko and V. P. Pishak, "Laser polarimetry of biological tissue: principles and applications," in Handbook of CoherentDomain Optical Methods: Biomedical Diagnostics, Environmental and Material Science, V. V. Tuchin, Ed., pp. 93-138, Kluwer Academic Publishers, Boston (2004).
3. V. V. Tuchin, "Polarized light interaction with tissues," J. Biomed. Opt. 21(7), 071114 (2016).

4. T. Novikova et al., "Polarimetric imaging for cancer diagnosis and staging," Opt. Photonics News 23(10), 26-33 (2012).

5. A. Pierangelo et al., "Polarimetric imaging of uterine cervix: a case study," Opt. Express 21(12), 14120-14130 (2013).

6. O. Peresunko, T. V. Kruk, and S. B. Yermolenko, "Diagnostic value spectropolarimetry of blood plasma in patients with breast cancer," Proc. SPIE 9166, 91661D (2014).

7. Y. A. Ushenko, "Investigation of formation and interrelations of polarization singular structure and Mueller-matrix images of biological tissues and diagnostics of their cancer changes," J. Biomed. Opt. 16(6), 066006 (2011).

8. E. Du et al., "Mueller matrix polarimetry for differentiating characteristic features of cancerous tissues," J. Biomed. Opt. 19(7), 076013 (2014).

9. Y. Yitzhaky, L. Graham, and I. Abdulhalim, "Analysis of skin moles from optical spectropolarimetric images," Proc. SPIE 8856, 88562J (2013).

10. T. Yasui, Y. Tohno, and T. Araki, "Characterization of collagen orientation in human dermis by two-dimensional second-harmonic-generation polarimetry," J. Biomed. Opt. 9(2), 259-264 (2004).

11. L. Graham, Y. Yitzhaky, and I. Abdulhalim, "Classification of skin moles from optical spectropolarimetric images: a pilot study," J. Biomed. Opt. 18(11), 111403 (2013).

12. K. M. Twietmeyer et al., "Mueller matrix retinal imager with optimized polarization conditions," Opt. Express 16(26), 21339-21354 (2008).

13. J. M. Bueno, "Measurement of parameters of polarization in the living human eye using imaging polarimetry," Vision Res. 40, 3791-3799 (2000).

14. R. Rawer, W. Stork, and K. D. Müller-Glaser, "Polarimetric methods for measurement of intra ocular glucose concentration," Biomed. Tech. 47, 186-188 (2002).

15. G. Purvinis, B. D. Cameron, and D. M. Altrogge, "Noninvasive polarimetric-based glucose monitoring: an in vivo study," J. Diabetes Sci. Technol. 5(2), 380-387 (2011).

16. P. Shukla and A. Pradhan, "Polarization-gated imaging in tissue phantoms: effect of size distribution," Appl. Opt. 48, 6099-6104 (2009).

17. B. Kunnen et al., "Application of circularly polarized light for noninvasive diagnosis of cancerous tissues and turbid tissue-like scattering media," J. Biophotonics 8(4), 317-323 (2015).

18. C. W. Sun et al., "Polarization gating in ultrafast-optics imaging of skeletal muscle tissues," Opt. Lett. 26(7), 432-434 (2001).

19. S. Sridhar and A. Da Silva, "Enhanced contrast and depth resolution in polarization imaging using elliptically polarized light," J. Biomed. Opt. 21(7), 071107 (2016).

20. S. L. Jacques, S. Roussel, and R. Samatham, "Polarized light imaging specifies the anisotropy of light scattering in the superficial layer of a tissue," J. Biomed. Opt. 21(7), 071115 (2016).

21. E. Garcia-Caurel et al., "Advanced Mueller ellipsometry instrumentation and data analysis," in Ellipsometry at Nanoscale, M. Losurdo and K. Hingerls, Eds., pp. 31-143, Springer-Verlag, Berlin (2013).

22. D. Goldstein, Polarized Light, 2nd ed., Marcel Dekker, New York (2003).

23. R. Ossikovski, "Differential and product Mueller matrix decompositions: a formal comparison," Opt. Lett. 37(2), 220-222 (2012).

24. R. Ossikovski, "Differential matrix formalism for depolarizing anisotropic media," Opt. Lett. 36(12), 2330-2332 (2011).

25. R. Ossikovski and O. Arteaga, "Statistical meaning of the differential Mueller matrix of depolarizing homogeneous media," Opt. Lett. 39(15), 4470-4473 (2014).

26. N. Ortega-Quijano and J. L. Arce-Diego, "Depolarizing differential Mueller matrices," Opt. Lett. 36(13), 2429-2431 (2011).

27. A. Peinado, A. Lizana, and J. Campos, "Optimization and tolerance analysis of a polarimeter with ferrolectric liquid crystals," Appl. Opt. 52, 5748-5757 (2013).

28. S. Alali, A. Gribble, and I. A. Vitkin, "Rapid wide-field Mueller matrix polarimetry imaging based on four photoelastic modulators with no moving parts," Opt. Lett. 41, 1038-1041 (2016).

29. A. Le Gratiet et al., "Scanning Mueller polarimetric microscopy," Opt. Lett. 41(18), 4336-4339 (2016). 
30. V. M. Turzhitsky et al., "Measuring mucosal blood supply in vivo with a polarization-gating probe," Appl. Opt. 47(32), 6046-6057 (2008).

31. M. P. Siege et al., "Assessment of blood supply in superficial tissue by polarization-gated elastic light-scattering spectroscopy," Appl. Opt. 45(2), 335-342 (2006).

32. Y. Liu, Y. L. Kim, and V. Backman, "Development of a bioengineered tissue model and its application in the investigation of the depth selectivity of polarization gating," Appl. Opt. 44(12), 2288-2299 (2005).

33. S. P. Morgan and M. Ridgway, "Polarization properties of light backscattered from a two layer scattering medium," Opt. Express 7(12), 395-402 (2000).

34. A. Da Silva, C. Deumié, and I. Vanzetta, "Elliptically polarized light for depth resolved optical imaging," Biomed. Opt. Express 3(11), 2907 (2012).

35. S. P. Morgan and I. M. Stockford, "Surface-reflection elimination in polarization imaging of superficial tissue," Opt. Lett. 28(2), 114-116 (2003).

36. J. S. Tyo et al., "Review of passive imaging polarimetry for remote sensing applications," Appl. Opt. 45(22), 5453-5469 (2006).

37. A. Peinado et al., "Optimization and performance criteria of a Stokes polarimeter based on two variable retarders," Opt. Express 18(10), 9815-9830 (2010).

38. R. A. Chipman, "Polarimetry," in Handbook of Optics, Chapter 22, pp. 1-37, McGraw-Hill, New York (1995).

39. H. J. van Staveren et al., "Light scattering in intralipid-10\% in the wavelength range of 400-1100 nm," Appl. Opt. 30(31), 4570-4514 (1991).

40. G. Anna et al., "Fully tunable active polarization imager for contrast enhancement and partial polarimetry," Appl. Opt. 51, 5302-5309 (2012).

41. F. Goudail, "Comparison of maximal achievable contrast in scalar, Stokes, and Mueller images," Opt. Lett. 35, 2600-2602 (2010).

42. K. Komatsu et al., "Polarized light microscopic analyses of collagen fibers in the rat incisor periodontal ligament in relation to areas, regions, and ages," Anat. Rec. 268(4), 381-387 (2002).

43. M. Egeblad, M. G. Rasch, and V. M. Weaver, "Dynamic interplay between the collagen scaffold and tumor evolution," Curr. Opin. Cell Biol. 22, 697-706 (2010).

44. M. W. Conklin et al., "Aligned collagen is a prognostic signature for survival in human breast carcinoma," Am. J. Pathol. 178, 1221-1232 (2011).

45. H. He et al., "A possible quantitative Mueller matrix transformation technique for anisotropic scattering media," Photonics Lasers Med. 2(2), 129-137 (2013).

46. J. J. Gil and R. Ossikovski, Polarized Light and the Mueller Matrix Approach, CRC Press, Taylor \& Francis, New York (2016).

47. F. H. Mustafa and M. S. Jaafar, "Comparison of wavelength-dependent penetration depths of lasers in different types of skin in photodynamic therapy," Indian J. Phys. 87(3), 203-209 (2013).

Angel Lizana received his MSc and $\mathrm{PhD}$ degrees in physics at the $U A B$ in 2006 and 2011, respectively. He was a postdoctoral scientist in the École Polytechnique, France, in 2012 to 2014. His research interests include liquid crystal displays and its application to diffractive optics, as well as the implementation of polarimeters and their use in biological applications. $\mathrm{He}$ is an associate professor at the UAB since 2016.

Albert Van Eeckhout received his BSc degree in physics at the University of Barcelona (UB) in 2015 and his MSc degree in photonics at the Polytechnique University of Barcelona (UPC), UB, and Autonomous University of Barcelona (UAB) in 2016. He is performing his $\mathrm{PhD}$ in physics at the UAB since 2017 and the topic of the thesis is related to the design and implementation of optical instrumentation based on polarization to be used in biomedical applications.

Kamil Adamczyk is a student at the University of Warsaw, Poland. He graduated with a bachelor's degree in physics and chemistry in 2015 and 2016. As part of his master's programme in physics at the same university, he participated in the exchange programme at the UAB. His academic interests revolve around experimental optics, in particular polarimetry and its use in biomedical applications.

Carla Rodríguez is a final year student of the physics degree at the UAB. She is interested in optics, and particularly, in polarimetry and its use in biomedical applications.

Juan C. Escalera received his degree in physics from the University of Zaragoza, Spain, in 1982 and his MS and PhD degrees from the UAB, Spain, in 1990 and 1991, respectively. He is a research collaborator with $U A B$ since 1988 and an associate professor with $U A B$ since 2001. His research interests include diffraction and color image formation, liquid crystal displays, and its applications in diffractive and polarization optics.

Enric Garcia-Caurel received his PhD in physics from the UB in 2001. He is a research associate at École Polytechnique, France, since 2003 and also at Synchrotron SOLEIL since 2015. His research includes ellipsometry and polarimetry for materials science, interaction of light in turbid media for biomedical optics, development of new optical instruments in collaboration with the industry, and, exploration of new applications of polarized light. He is a senior member of SPIE.

Ignacio Moreno received his $\mathrm{BS}$ and $\mathrm{PhD}$ degrees in physics at the UAB in 1992 and 1996, respectively. He is a full professor of optics at the University Miguel Hernandez of Elche. He is currently the president of the Spanish Optical Society-SEDOPTICA. He is a fellow member of SPIE and OSA. His research interests include liquid crystal displays and its applications in diffractive and polarization optics.

Juan Campos received his MSc degree in physics at the University of Zaragoza in 1981 and his $\mathrm{PhD}$ at the $\mathrm{UAB}$ in 1986 . He was a professor of optics at the UB. From 1993, he was a professor at the UAB and since 2005 he is a full professor. He has worked in image processing, pattern recognition, digital holography, color information, liquid crystals, surface metrology, and polarimetry. He is a fellow of the SPIE, OSA, and EOS. 